\title{
Hölder regularity for porous medium systems
}

\author{
Naian Liao $^{1}$
}

Received: 24 June 2020 / Accepted: 16 May 2021 / Published online: 14 July 2021

(c) The Author(s) 2021

\section{Abstract}

We establish Hölder continuity for locally bounded weak solutions to certain parabolic systems of porous medium type, i.e.

$$
\partial_{t} \mathbf{u}-\operatorname{div}\left(m|\mathbf{u}|^{m-1} D \mathbf{u}\right)=0, \quad m>0 .
$$

As a consequence of our local Hölder estimates, a Liouville type result for bounded global solutions is also established. In addition, sufficient conditions are given to ensure local boundedness of local weak solutions.

Mathematics Subject Classification $35 \mathrm{~K} 40 \cdot 35 \mathrm{~K} 65 \cdot 35 \mathrm{~K} 67 \cdot 35 \mathrm{~B} 65 \cdot 35 \mathrm{~B} 45$

\section{Introduction and main results}

Let $E$ be an open set in $\mathbb{R}^{N}$ and $E_{T}:=E \times(0, T]$ for some $T>0$. For a positive $m$, we are interested in the following parabolic system

$$
\left\{\begin{array}{l}
\partial_{t} u_{i}-\operatorname{div}\left(m|\mathbf{u}|^{m-1} D u_{i}\right)=0 \quad \text { weakly in } E_{T}, \\
i=1, \cdots, n, \quad \mathbf{u} \equiv\left(u_{1}, \cdots, u_{n}\right) .
\end{array}\right.
$$

In the scalar case, i.e. $n=1$, it is commonly referred to as the porous medium equation. It presents degeneracy in the set $[|\mathbf{u}|=0]$ when $m>1$ and singularity when $0<m<1$.

As is well known, everywhere regularity is in general not expected from systems of elliptic or parabolic type (cf. [8]). Nevertheless, the special quasi-diagonal structure of (I) enables us to achieve the Hölder regularity of locally bounded solutions.

Let $\mathbf{u}$ be a solution to the system (I). Loosely speaking, our main result reads

$$
\mathbf{u} \in L_{\mathrm{loc}}^{\infty}\left(E_{T}\right) \Longrightarrow \mathbf{u} \in C_{\mathrm{loc}}^{\alpha}\left(E_{T}\right)
$$

for some $\alpha \in(0,1)$ depending only on $n, N$ and the exponent $m$; see Theorem 1.1 for details. Based on our explicit local estimates, we are able to obtain a Liouville type result, which

Communicated by A. Malchiodi.

$\bowtie$ Naian Liao

naian.liao@sbg.ac.at

1 Fachbereich Mathematik, Universität Salzburg, Hellbrunner Str. 34, 5020 Salzburg, Austria 
asserts bounded global solutions must be constant; see Corollary 1.1. In addition, we explore sufficient conditions that ensure local boundedness of solutions in Sect. 6.

The norm of $\mathbf{u}$ refers to the standard Euclidean norm, i.e. $|\mathbf{u}|=\left(u_{1}^{2}+u_{2}^{2}+\cdots+u_{n}^{2}\right)^{\frac{1}{2}}$. However, it is worth pointing out that our Hölder estimate for solutions to the system (I) still holds for any norm of $\mathbf{u}$. This point will be clear from the proof. Thus, in particular, we could use $|\mathbf{u}|_{1}=\left|u_{1}\right|+\left|u_{2}\right|+\cdots+\left|u_{n}\right|$ in the system (I). Moreover, our results also hold for more general systems modeled on (I), which permit us to insert bounded and measurable diffusion coefficients in the system (I) (cf. (1.1) - (1.2)).

Our motivation of studying the system (I) with measurable coefficients as in (1.1) - (1.2) mainly stems from the $C^{1, \alpha}$ regularity for the parabolic $p$-Laplace equation, i.e.

$$
u_{t}-\operatorname{div}\left(|D u|^{p-2} D u\right)=0 .
$$

The spatial gradient $D u$ will satisfy a system with structures similar to (I). In fact, we may differentiate the above equation in $x_{s}$ formally to obtain

$$
u_{t x_{s}}-\left(|D u|^{p-2} u_{x_{j} x_{s}}+(p-2)|D u|^{p-2} \frac{u_{x_{i}} u_{x_{j}}}{|D u|^{2}} u_{x_{i} x_{s}}\right)_{x_{j}}=0 .
$$

Here the summation convention is evoked. Introduce the vector valued function $\mathbf{v}:=D u$. In terms of $\mathbf{v}$ the above differentiated equation can be written as

$$
v_{s, t}-\left(a_{i j}|\mathbf{v}|^{p-2} v_{s, x_{i}}\right)_{x_{j}}=0, \quad s=1, \cdots, N,
$$

where the measurable coefficients are defined by

$$
a_{i j}=\delta_{i j}+(p-2) \frac{v_{i} v_{j}}{|\mathbf{v}|^{2}},
$$

which in turn fulfills the condition

$$
\min \{p-1,1\}|\xi|^{2} \leq a_{i j} \xi_{i} \xi_{j} \leq \max \{p-1,1\}|\xi|^{2} \quad \text { for any } \xi \in \mathbb{R}^{N} .
$$

In particular, when $N=1, \mathbf{v}$ reduces to a scalar function $v$ that satisfies the one dimensional porous medium equation

$$
v_{t}-(p-1)\left(|v|^{p-2} v_{x}\right)_{x}=0 .
$$

Therefore $D u$ satisfies a system with the same structure as (I) for $m=p-1$; see the general system (1.1) - (1.2). Particularly, our main result implies the following well-known $C^{1, \alpha}$ regularity of a weak solution $u$ to the parabolic $p$-Laplace equation (cf. [2-4,7,17]):

$$
D u \in L_{\mathrm{loc}}^{\infty} \Longrightarrow D u \in C_{\mathrm{loc}}^{\alpha} .
$$

Our approach to the Hölder regularity for (I) is inspired by [5] where the $C^{1, \alpha}$ regularity for the elliptic $p$-Laplace equation has been investigated. The proof in [5] reflects a general idea due to De Giorgi: if the set where the equation is degenerate is confined within a small portion of a ball, then it is actually non-degenerate (cf. Sect. 3); if conversely the degenerate set occupies a seizable portion, then the solution can be compared with the radius of the ball (cf. Sect. 4).

The main new input of our argument lies in the introduction of proper intrinsically scaled cylinders, instead of balls, where the homogeneity of the parabolic systems is restored. We refer to $[4,6]$ for an account of the theory for intrinsic scaling. It would also be helpful to refer to the recent work [14] on the Hölder regularity for porous medium type equations. Some arguments we use here are closely related to this work. This kind of idea has been used in [4,7] 
to treat the $C^{1, \alpha}$ regularity for parabolic systems of $p$-Laplacian type, via a combination of Campanato's approach and De Giorgi's iteration. Nevertheless, the quasi-diagonal structures of the system (I) allow us to implement the idea of intrinsic scaling more or less like we deal with scalar equations. Once proper energy estimates are derived from the system, we do not refer back to it any more. Thus we rely solely on De Giorgi's techniques.

Finally, we mention that there is a "cousin" of the system (I), namely

$$
\left\{\begin{array}{l}
\partial_{t} u_{i}-\Delta\left(|\mathbf{u}|^{m-1} u_{i}\right)=0 \quad \text { weakly in } E_{T}, \\
i=1, \cdots, n, \quad \mathbf{u} \equiv\left(u_{1}, \cdots, u_{n}\right) .
\end{array}\right.
$$

The systems (I) and (II) are formally identical only in the scalar case, i.e. $n=1$. The system (II) has been derived in [19] from Bean's critical-state model in the superconductivity theory. With the norm $|\mathbf{u}|_{1}$, it has been established in [11] modeling a competitive adsorption process among different species, via a multi-component isotherm of Freundlich type.

A study of Hölder regularity is carried out in [11] for non-negative solutions to (II) with the norm $|\mathbf{u}|_{1}$. The non-negativity of $\mathbf{u}$ turns out to be essential, since the argument relies on the fact that $|\mathbf{u}|_{1}$ satisfies the scalar porous medium equation. See [11,19] for discussions on Dirichlet problems and $[9,16]$ on Cauchy problems.

Similar interest in the Hölder regularity of solutions to various parabolic systems also appears in $[13,15,18]$. Certain degeneracy is considered in [13] for systems with different structures from ours, whereas systems in $[15,18]$ are non-degenerate as the arguments are based on the regularity criterion in [8].

\subsection{Main results}

We postpone the formal definition of weak solutions to Sect. 1.3 and refer to Sect. 1.2 for the use of notations. Now we proceed to state in a precise manner our main results concerning the interior Hölder continuity of weak solutions to the system (I).

Theorem 1.1 Let $\mathbf{u}$ be a bounded, local, weak solution to the system (I) in $E_{T}$. Then $\mathbf{u}$ is locally Hölder continuous in $E_{T}$. More precisely, setting $M:=\|\mathbf{u}\|_{\infty, E_{T}}$, there exist constants $\gamma>1$ and $\alpha \in(0,1)$ that can be determined a priori only in terms of $m, n$ and $N$, such that for every compact set $\mathcal{K} \subset E_{T}$,

$$
\begin{aligned}
& \left|\mathbf{u}\left(x_{1}, t_{1}\right)-\mathbf{u}\left(x_{2}, t_{2}\right)\right| \leq \gamma M\left(\frac{\left|x_{1}-x_{2}\right|+M^{\frac{m-1}{2}}\left|t_{1}-t_{2}\right|^{\frac{1}{2}}}{\operatorname{dist}_{1}(\mathcal{K} ; \Gamma)}\right)^{\alpha}, \quad m>1, \\
& \left|\mathbf{u}\left(x_{1}, t_{1}\right)-\mathbf{u}\left(x_{2}, t_{2}\right)\right| \leq \gamma M\left(\frac{M^{\frac{1-m}{2}}\left|x_{1}-x_{2}\right|+\left|t_{1}-t_{2}\right|^{\frac{1}{2}}}{\operatorname{dist}_{2}(\mathcal{K} ; \Gamma)}\right)^{\alpha}, \quad 0<m<1,
\end{aligned}
$$

for every pair of points $\left(x_{1}, t_{1}\right),\left(x_{2}, t_{2}\right) \in \mathcal{K}$. Here dist $_{1}$ and dist $_{2}$ are intrinsic distances from $\mathcal{K}$ to the parabolic boundary $\Gamma$ of $E_{T}$ defined in Sect. 1.2.

Remark 1.1 We have stated Theorem 1.1 for globally bounded weak solutions. However the proof has a local thrust. As a matter of fact, for any $M>0$ verifying

$$
\begin{aligned}
& \max _{1 \leq i \leq n}^{\operatorname{ess} \text { osc }} u_{i} \leq M, \quad \text { where } \theta=M^{1-m}, \quad m>1, \\
& \max _{1 \leq i \leq n\left(Q_{\varrho}(\theta)\right.}^{\operatorname{ess} \text { osc }} u_{\left(x_{o}\right)+\mathcal{Q}_{\varrho}(\vartheta)} u_{i} \leq M, \quad \text { where } \vartheta=M^{\frac{m-1}{2}}, \quad 0<m<1,
\end{aligned}
$$


for some cylinders $\left(x_{o}, t_{o}\right)+Q_{\varrho}(\theta)$ and $\left(x_{o}, t_{o}\right)+\mathcal{Q}_{\varrho}(\vartheta)$ included in $E_{T}$, we will show the following oscillation decay for $0<r<\varrho$ :

$$
\begin{aligned}
& \max _{1 \leq i \leq n} \underset{\left(x_{o}, t_{o}\right)+Q_{r}(\theta)}{\operatorname{ess} \text { osc }} u_{i} \leq \gamma M\left(\frac{r}{\varrho}\right)^{\alpha}, \quad m>1, \\
& \max _{1 \leq i \leq n\left(x_{o}, t_{o}\right)+\mathcal{Q}_{r}(\vartheta)}^{\operatorname{ess} \text { osc }} u_{i} \leq \gamma M\left(\frac{r}{\varrho}\right)^{\alpha}, \quad 0<m<1 .
\end{aligned}
$$

See Sect. 1.2 for the meaning of these cylinders. The conclusion of Theorem 1.1 can be derived from this oscillation estimate via a standard covering argument.

Remark 1.2 Theorem 1.1 continues to hold for systems with more general structure modeled on (I); see (1.1) - (1.2). As a result, the constants $\alpha$ and $\gamma$ also depend on the structural constants $C_{o}$ and $C_{1}$ in (1.2).

The oscillation decay in Remark 1.1, while local in nature, has a global implication. Indeed, let $\mathbf{u}$ be a bounded, local weak solution to the system (I) in the semi-infinite strip $\mathcal{S}_{T}:=\mathbb{R}^{N} \times(-\infty, T)$ for some $T \in \mathbb{R}$. Then when $m>1$, we have for $0<r<\varrho$ that

$$
\max _{1 \leq i \leq n} \underset{\left(x_{o}, t_{o}\right)+Q_{r}(\theta)}{\operatorname{ess} \operatorname{osc}} u_{i} \leq \gamma\|\mathbf{u}\|_{\infty, \mathcal{S}_{T}}\left(\frac{r}{\varrho}\right)^{\alpha},
$$

for any cylinder $\left(x_{o}, t_{o}\right)+Q_{r}(\theta)$ compactly included in $\mathcal{S}_{T}$. Now fixing $r$ and letting $\varrho \rightarrow \infty$, we immediately arrive at a Liouville-type result. The case $0<m<1$ can be analyzed similarly. Thus we arrive at the following Liouville type result.

Corollary 1.1 A bounded, local weak solution to the system (I) in $\mathcal{S}_{T}$ must be a constant.

Remark 1.3 Unlike elliptic equations, one-sided boundedness of solutions in $\mathcal{S}_{T}$ is generally not sufficient to imply solutions to parabolic equations are constants. This is evident from the non-negative solution $u(x, t)=e^{x+t}$ to the one dimensional heat equation. See [6, p. 105] for more on Liouville type results of parabolic equations.

Remark 1.4 The global boundedness condition in Corollary 1.1 can be easily relaxed to allow $|\mathbf{u}|$ to grow slower than $\left(|x|+|t|^{\frac{1}{2}}\right)^{\alpha}$ as $|x| \rightarrow \infty$ and $t \rightarrow-\infty$.

\subsection{Notations}

Suppose $\mathbf{u}$ is a bounded solution to (I) in $E_{T}$. Let $\Gamma:=\partial E_{T}-\bar{E} \times\{T\}$ be the parabolic boundary of $E_{T}$, and for a compact set $\mathcal{K} \subset E_{T}$ introduce the intrinsic, parabolic $m$-distance from $\mathcal{K}$ to $\Gamma$ by

$$
\begin{aligned}
& \operatorname{dist}_{1}(\mathcal{K} ; \Gamma):=\inf _{\substack{(x, t) \in \mathcal{K} \\
(y, s) \in \Gamma}}\left\{|x-y|+\|\mathbf{u}\|_{\infty}^{\frac{m-1}{2}}|t-s|^{\frac{1}{2}}\right\}, \quad m>1, \\
& \operatorname{dist}_{2}(\mathcal{K} ; \Gamma):=\inf _{\substack{(x, t) \in \mathcal{K} \\
(y, s) \in \Gamma}}\left\{\|\mathbf{u}\|_{\infty}^{\frac{1-m}{2}}|x-y|+|t-s|^{\frac{1}{2}}\right\}, \quad 0<m<1 .
\end{aligned}
$$

For $\varrho>0$ let $K_{\varrho}\left(x_{o}\right)$ be the cube with center at $x_{o} \in \mathbb{R}^{N}$ and edge $2 \varrho$. We define backward cylinders scaled by a positive parameter $\theta$ by

$$
\left(x_{o}, t_{o}\right)+Q_{\varrho}(\theta):=K_{\varrho}\left(x_{o}\right) \times\left(t_{o}-\theta \varrho^{2}, t_{o}\right] .
$$


We also need another type of cylinders

$$
\left(x_{o}, t_{o}\right)+\mathcal{Q}_{\varrho}(\vartheta):=K_{\vartheta \varrho}\left(x_{o}\right) \times\left(t_{o}-\varrho^{2}, t_{o}\right],
$$

for some positive parameter $\vartheta$. In Sect. 6, we will use the following type of cylinders

$$
\left(x_{o}, t_{o}\right)+Q_{\varrho, \tau}:=K_{\varrho}\left(x_{o}\right) \times\left(t_{o}-\tau, t_{o}\right] .
$$

When $\left(x_{o}, t_{o}\right)=(0,0)$ or $\theta=\vartheta=1$, we will omit them from the notation.

In what follows, we will use $\gamma$ as a generic positive constant in our estimates, which may change from line to line. Nevertheless, such $\gamma$ can be determined a priori in terms of given data.

\subsection{Notion of solution}

It is always very important to pinpoint what we mean by a weak solution, as different notions may lead to different implications. In the following we may consider a general system modeled on the system (I):

$$
\left\{\begin{array}{l}
\partial_{t} u_{i}-\operatorname{div} \mathbf{A}^{(i)}(x, t, \mathbf{u}, D \mathbf{u})=0 \quad \text { weakly in } E_{T} \\
i=1, \cdots, n, \quad \mathbf{u} \equiv\left(u_{1}, \cdots, u_{n}\right)
\end{array}\right.
$$

where the functions

$$
\mathbf{A}^{(i)}:=\left(A_{1}^{(i)}, \cdots, A_{N}^{(i)}\right): E_{T} \times \mathbb{R}^{N} \times \mathbb{R}^{n N} \rightarrow \mathbb{R}^{N}
$$

are Carathéodory, i.e. $A_{j}^{(i)}(x, t, \mathbf{u}, \mathbf{z})$ is measurable in $(x, t)$ for all $(\mathbf{u}, \mathbf{z}) \in \mathbb{R}^{N} \times \mathbb{R}^{n N}$ and continuous in $(\mathbf{u}, \mathbf{z})$ for a.e. $(x, t) \in E_{T}$, and satisfy the structure conditions

$$
\left\{\begin{array}{l}
\mathbf{A}^{(i)}(x, t, \mathbf{u}, D \mathbf{u}) \cdot D u_{i} \geq C_{o}|\mathbf{u}|^{m-1}\left|D u_{i}\right|^{2} \\
\left|\mathbf{A}^{(i)}(x, t, \mathbf{u}, D \mathbf{u})\right| \leq C_{1}|\mathbf{u}|^{m-1}\left|D u_{i}\right|
\end{array}\right.
$$

for all $i=1, \cdots, n$ and for some positive $m, C_{o}$ and $C_{1}$. Together with $n$ and $N$, they will be referred to as the data in the sequel.

A vector valued function $\mathbf{u}$ satisfying

$$
\begin{cases}u_{i} \in C\left(0, T ; L_{\mathrm{loc}}^{2}(E)\right),|\mathbf{u}|^{m-1} u_{i}, u_{i} \in L_{\mathrm{loc}}^{2}\left(0, T ; W_{\mathrm{loc}}^{1,2}(E)\right), & m>1, \\ u_{i} \in C\left(0, T ; L_{\mathrm{loc}}^{m+1}(E)\right),|\mathbf{u}|^{m-1} u_{i},\left|u_{i}\right|^{m-1} u_{i} \in L_{\mathrm{loc}}^{2}\left(0, T ; W_{\mathrm{loc}}^{1,2}(E)\right), 0<m<1,\end{cases}
$$

is a local, weak sub(super)-solution to (1.1) with the structure conditions (1.2), if for every compact set $K \subset E$ and every sub-interval $\left[t_{1}, t_{2}\right] \subset(0, T]$,

$$
\left.\int_{K} u_{i} \phi \mathrm{d} x\right|_{t_{1}} ^{t_{2}}+\iint_{K \times\left(t_{1}, t_{2}\right)}\left[-u_{i} \phi_{t}+\mathbf{A}^{(i)}(x, t, \mathbf{u}, D \mathbf{u}) \cdot D \phi\right] \mathrm{d} x \mathrm{~d} t \leq(\geq) 0
$$

for all non-negative test functions

$$
\phi \in W_{\mathrm{loc}}^{1,2}\left(0, T ; L^{2}(K)\right) \cap L_{\mathrm{loc}}^{2}\left(0, T ; W_{o}^{1,2}(K)\right) .
$$

Remark 1.5 The notion of solution may generate discrepancies, as it is not entirely clear even in the scalar case. An interesting study on the equivalence of different notions of solution for the prototype porous medium equation is carried out in [1]. 
In what follows, special care needs to be taken for the time variable of a solution since we will use test functions that involve the solution itself. Lack of regularity in the time variable notwithstanding, they become admissible provided we employ a proper mollification in the time variable.

To this end, we introduce for any $v \in L^{1}\left(E_{T}\right)$,

$$
\llbracket v \rrbracket_{h}(x, t):=\frac{1}{h} \int_{0}^{t} \mathrm{e}^{\frac{s-t}{h}} v(x, s) \mathrm{d} s, \quad \llbracket v \rrbracket_{h}(x, t):=\frac{1}{h} \int_{t}^{T} \mathrm{e}^{\frac{t-s}{h}} v(x, s) \mathrm{d} s .
$$

Properties of this mollification can for instance be found in [10, Lemma 2.2]. In particular, we need the following identities:

$$
\partial_{t} \llbracket v \rrbracket_{h}=\frac{1}{h}\left(v-\llbracket v \rrbracket_{h}\right), \quad \partial_{t} \llbracket v \rrbracket_{\bar{h}}=\frac{1}{h}\left(\llbracket v \rrbracket_{\bar{h}}-v\right) .
$$

From the weak form (1.3) of the differential inequality for sub(super)-solutions we may deduce the mollified version:

$$
\begin{gathered}
\iint_{E_{T}}\left[\partial_{t} \llbracket u_{i} \rrbracket_{h} \phi+\llbracket \mathbf{A}^{(i)}(x, t, \mathbf{u}, D \mathbf{u}) \rrbracket_{h} \cdot D \phi\right] \mathrm{d} x \mathrm{~d} t \\
\quad \leq(\geq) \int_{E} u_{i}(x, 0) \cdot \frac{1}{h} \int_{0}^{T} \mathrm{e}^{-\frac{s}{h}} \phi(x, s) \mathrm{d} s \mathrm{~d} x,
\end{gathered}
$$

for any non-negative test function $\phi \in L^{p}\left(0, T ; W_{0}^{1, p}(E)\right)$ with compact support in $E_{T}$. Indeed, we may take the test function $\llbracket \phi \rrbracket_{\bar{h}}$ in the weak formulation (1.3) of sub(super)solutions to (I). Since $\phi$ is compactly supported in $E_{T}$, we have after an application of Fubini's theorem

$$
\begin{aligned}
& \iint_{E_{T}}\left[-u_{i} \partial_{t} \llbracket \phi \rrbracket_{\bar{h}}+\llbracket \mathbf{A}^{(i)}(x, t, \mathbf{u}, D \mathbf{u}) \rrbracket_{h} \cdot D \phi\right] \mathrm{d} x \mathrm{~d} t \\
& \quad \leq(\geq) \int_{E} u_{i}(x, 0) \cdot \frac{1}{h} \int_{0}^{T} \mathrm{e}^{-\frac{s}{h}} \phi(x, s) \mathrm{d} s \mathrm{~d} x .
\end{aligned}
$$

On the other hand, we calculate the term involving the time derivative using (1.4)

$$
\begin{aligned}
\iint_{E_{T}} u_{i} \partial_{t} \llbracket \phi \rrbracket_{h} \mathrm{~d} x \mathrm{~d} t & =\frac{1}{h} \iint_{E_{T}} u_{i}\left(\llbracket \phi \rrbracket_{h}-\phi\right) \mathrm{d} x \mathrm{~d} t \\
& =\frac{1}{h} \iint_{E_{T}}\left(\llbracket u_{i} \rrbracket_{h}-u_{i}\right) \phi \mathrm{d} x \mathrm{~d} t=-\iint_{E_{T}} \partial_{t} \llbracket u_{i} \rrbracket_{h} \phi \mathrm{d} x \mathrm{~d} t .
\end{aligned}
$$

This yields the desired claim in (1.5).

\section{Energy estimates}

This section is devoted to local energy estimates for the general system (1.1) - (1.2). Due to the degenerate or singular nature of the system, we need to consider the case $0<m<1$ (Proposition 2.1) and the case $m>1$ (Proposition 2.2) separately.

Proposition 2.1 Let $0<m<1$ and $\mathbf{u}$ be a local weak sub(super)-solution to (1.1) - (1.2) in $E_{T}$. There exists a constant $\gamma>0$, such that for all cylinders $Q_{R, S}:=K_{R}\left(x_{o}\right) \times\left(t_{o}-S, t_{o}\right] \subset$ $E_{T}$, every $k \in \mathbb{R}$, every $i \in\{1, \cdots, n\}$, and every non-negative, piecewise smooth cutoff 
function $\zeta$ in $Q_{R, S}$ vanishing on $\partial K_{R}\left(x_{o}\right)$,

$$
\begin{aligned}
& \underset{t_{o}-S<t<t_{o}}{\operatorname{ess} \sup _{K_{R}\left(x_{o}\right)}} \int_{ \pm}\left(u_{i}, k\right) \zeta^{2}(x, t) \mathrm{d} x+\iint_{Q_{R, S}}|\mathbf{u}|^{2(m-1)}\left|D\left(u_{i}-k\right)_{ \pm}\right|^{2} \zeta^{2} \mathrm{~d} x \mathrm{~d} t \\
& \quad \leq \gamma \iint_{Q_{R, S}}\left(u_{i}-k\right)_{ \pm}^{2 m}|D \zeta|^{2} \mathrm{~d} x \mathrm{~d} t+\gamma \iint_{Q_{R, S}}\left(u_{i}-k\right)_{ \pm}^{1+m}\left|\zeta_{t}\right| \mathrm{d} x \mathrm{~d} t \\
& \quad+\int_{K_{R}\left(x_{o}\right)} \mathfrak{g}_{ \pm}\left(u_{i}, k\right) \zeta^{2}\left(x, t_{o}-S\right) \mathrm{d} x
\end{aligned}
$$

where we have defined

$$
\mathfrak{g}_{ \pm}\left(u_{i}, k\right):= \pm \int_{k}^{u_{i}}(\varphi(s)-\varphi(k))_{ \pm} \mathrm{d} s, \quad \varphi(s):=|s|^{m-1} s .
$$

Proof We prove the statement for sub-solutions only, as the other case is similar. For fixed $t_{o}-S<t_{1}<t_{2}<t_{o}$ and $\varepsilon>0$ small enough we define the cutoff function in time by

$$
\psi_{\varepsilon}(t):=\left\{\begin{array}{cl}
0, & \text { for } t_{o}-S \leq t \leq t_{1}-\varepsilon, \\
1+\frac{t-t_{1}}{\varepsilon}, & \text { for } t_{1}-\varepsilon<t \leq t_{1}, \\
1, & \text { for } t_{1}<t \leq t_{2} \\
1-\frac{t-t_{2}}{\varepsilon}, & \text { for } t_{2}<t \leq t_{2}+\varepsilon, \\
0, & \text { for } t_{2}+\varepsilon<t \leq t_{o} .
\end{array}\right.
$$

Now, we choose in (1.5) the test function

$$
Q_{R, S} \ni(x, t) \mapsto \phi(x, t)=\zeta^{2}(x, t) \psi_{\varepsilon}(t)\left(\varphi\left(u_{i}(x, t)\right)-\varphi(k)\right)_{+} .
$$

It is an admissible test function thanks to the notion of solution and the choice of cutoff functions. In the following we omit the reference to the vertex $\left(x_{o}, t_{o}\right)$ in the notation.

For the integral in (1.5) containing the time derivative we compute

$$
\begin{aligned}
& \iint_{E_{T}} \partial_{t} \llbracket u_{i} \rrbracket_{h} \phi \mathrm{d} x \mathrm{~d} t=\iint_{Q_{R, S}} \zeta^{2} \psi_{\varepsilon} \partial_{t} \llbracket u_{i} \rrbracket_{h}\left(\varphi\left(\llbracket u_{i} \rrbracket_{h}\right)-\varphi(k)\right)_{+} \mathrm{d} x \mathrm{~d} t \\
& \quad+\iint_{Q_{R, S}} \zeta^{2} \psi_{\varepsilon} \partial_{t} \llbracket u_{i} \rrbracket_{h}\left[\left(\varphi\left(u_{i}(x, t)\right)-\varphi(k)\right)_{+}-\left(\varphi\left(\llbracket u_{i} \rrbracket_{h}\right)-\varphi(k)\right)_{+}\right] \mathrm{d} x \mathrm{~d} t \\
& \quad \geq \iint_{Q_{R, S}} \zeta^{2} \psi_{\varepsilon} \partial_{t} \mathfrak{g}_{+}\left(\llbracket u_{i} \rrbracket_{h}, k\right) \mathrm{d} x \mathrm{~d} t \\
& \quad=-\iint_{Q_{R, S}}\left(\zeta^{2} \psi_{\varepsilon}^{\prime}+\psi_{\varepsilon} \partial_{t} \zeta^{2}\right) \mathfrak{g}_{+}\left(\llbracket u_{i} \rrbracket_{h}, k\right) \mathrm{d} x \mathrm{~d} t .
\end{aligned}
$$

Here we have used in the second line the identity (1.4) $)_{1}$ and the fact that the map $s \mapsto$ $(\varphi(s)-\varphi(k))_{+}$is a monotone increasing function, which implies that the term in the second line is non-negative. Since $\llbracket u_{i} \rrbracket_{h} \rightarrow u_{i}$ in $L^{2}\left(\Omega_{T}\right)$ (cf. [10, Lemma 2.2]), we can pass to the limit as $h \rightarrow 0$ in the integral on the right-hand side. We therefore get

$$
\begin{aligned}
\liminf _{h \rightarrow 0} \iint_{Q_{R, S}} \partial_{t} \llbracket u_{i} \rrbracket_{h} \phi \mathrm{d} x \mathrm{~d} t & \geq-\iint_{Q_{R, S}}\left(\zeta^{2} \psi_{\varepsilon}^{\prime}+\psi_{\varepsilon} \partial_{t} \zeta^{2}\right) \mathfrak{g}_{+}\left(u_{i}, k\right) \mathrm{d} x \mathrm{~d} t \\
& =:-\left[\mathbf{I}_{\varepsilon}+\mathbf{I I}_{\varepsilon}\right],
\end{aligned}
$$

with the obvious meaning of $\mathbf{I}_{\varepsilon}$ and $\mathbf{I I}_{\varepsilon}$. We now pass to the limit $\varepsilon \rightarrow 0$. For the term $\mathbf{I}_{\varepsilon}$ we obtain for any $t_{o}-S<t_{1}<t_{2}<t_{o}$ that

$$
\lim _{\varepsilon \rightarrow 0} \mathbf{I}_{\varepsilon}=\int_{K_{R}} \zeta^{2}\left(x, t_{1}\right) \mathfrak{g}_{+}\left(u_{i}\left(x, t_{1}\right), k\right) \mathrm{d} x-\int_{K_{R}} \zeta^{2}\left(x, t_{2}\right) \mathfrak{g}_{+}\left(u_{i}\left(x, t_{2}\right), k\right) \mathrm{d} x,
$$


whereas for $\mathbf{I I}_{\varepsilon}$ we have

$$
\lim _{\varepsilon \rightarrow 0} \mathbf{I I}_{\varepsilon}=\iint_{K_{R} \times\left(t_{1}, t_{2}\right)} \partial_{t} \zeta^{2} \mathfrak{g}_{+}\left(u_{i}, k\right) \mathrm{d} x \mathrm{~d} t .
$$

Next, we observe that the term on the right-hand side of (1.5) disappears as $h \rightarrow 0$, since by construction $\phi(\cdot, 0) \equiv 0$ on $E$, i.e. we have

$$
\lim _{h \rightarrow 0} \int_{E} u_{i}(x, 0) \cdot \frac{1}{h} \int_{0}^{T} \mathrm{e}^{-\frac{s}{h}} \phi(x, s) \mathrm{d} s \mathrm{~d} x=\int_{E} u_{i}(x, 0) \phi(x, 0) \mathrm{d} x=0 .
$$

It remains to consider the diffusion term. After passing to the limit as $h \rightarrow 0$, we use the structure conditions (1.2) for the vector-field $\mathbf{A}^{(i)}$, and subsequently Young's inequality to estimate

$$
\begin{aligned}
\lim _{h \rightarrow 0} & \iint_{E_{T}} \llbracket \mathbf{A}^{(i)}(x, t, \mathbf{u}, D \mathbf{u}) \rrbracket_{h} \cdot D \phi \mathrm{d} x \mathrm{~d} t \\
= & \iint_{Q_{R, S}} \psi_{\varepsilon} \mathbf{A}^{(i)}(x, t, \mathbf{u}, D \mathbf{u}) \cdot\left[\zeta^{2} D\left(\varphi\left(u_{i}\right)-\varphi(k)\right)_{+}\right. \\
& \left.+2 \zeta\left(\varphi\left(u_{i}\right)-\varphi(k)\right)_{+} D \zeta\right] \mathrm{d} x \mathrm{~d} t \\
\geq & C_{O} \iint_{Q_{R, S}} \psi_{\varepsilon}|\mathbf{u}|^{2(m-1)}\left|D\left(u_{i}-k\right)_{+}\right|^{2} \zeta^{2} \mathrm{~d} x \mathrm{~d} t \\
& -2 C_{1} \iint_{Q_{R, S}} \zeta|D \zeta| \psi_{\varepsilon}|\mathbf{u}|^{(m-1)}\left(\varphi\left(u_{i}\right)-\varphi(k)\right)_{+}\left|D\left(u_{i}-k\right)_{+}\right| \mathrm{d} x \mathrm{~d} t \\
\geq & \frac{C_{O}}{2} \iint_{Q_{R, S}} \psi_{\varepsilon}|\mathbf{u}|^{2(m-1)}\left|D\left(u_{i}-k\right)_{+}\right|^{2} \zeta^{2} \mathrm{~d} x \mathrm{~d} t \\
\quad & -\gamma \iint_{Q_{R, S}} \psi_{\varepsilon}\left(\varphi\left(u_{i}\right)-\varphi(k)\right)_{+}^{2}|D \zeta|^{2} \mathrm{~d} x \mathrm{~d} t .
\end{aligned}
$$

Combining the preceding estimates and letting $\varepsilon \rightarrow 0$ we arrive at

$$
\begin{aligned}
& \int_{K_{R} \times\left\{t_{2}\right\}} \zeta^{2} \mathfrak{g}_{+}\left(u_{i}, k\right) \mathrm{d} x+\frac{C_{o}}{2} \iint_{K_{R} \times\left(t_{1}, t_{2}\right)}|\mathbf{u}|^{2(m-1)}\left|D\left(u_{i}-k\right)_{+}\right|^{2} \zeta^{2} \mathrm{~d} x \mathrm{~d} t \\
& \quad \leq \gamma \iint_{K_{R} \times\left(t_{1}, t_{2}\right)}\left[\left(\varphi\left(u_{i}\right)-\varphi(k)\right)_{+}^{2}|D \zeta|^{2}+\partial_{t} \zeta^{2} \mathfrak{g}_{+}\left(u_{i}, k\right)\right] \mathrm{d} x \mathrm{~d} t \\
& \quad+\int_{K_{R} \times\left\{t_{1}\right\}} \zeta^{2} \mathfrak{g}_{+}\left(u_{i}, k\right) \mathrm{d} x,
\end{aligned}
$$

whenever $t_{o}-S<t_{1}<t_{2}<t_{o}$. Remembering $0<m<1$, the terms in the bracket are estimated by

$$
\left(\varphi\left(u_{i}\right)-\varphi(k)\right)_{+} \leq\left(u_{i}-k\right)_{+}^{m},
$$

and

$$
\mathfrak{g}_{+}\left(u_{i}, k\right)=\int_{k}^{u_{i}}(\varphi(s)-\varphi(k))_{+} \mathrm{d} s \leq \int_{k}^{u_{i}}(s-k)_{+}^{m} \mathrm{~d} s=\frac{1}{1+m}\left(u_{i}-k\right)_{+}^{1+m} .
$$

Recalling $u_{i} \in C\left(0, T ; L_{\text {loc }}^{m+1}(E)\right)$, then one easily obtains the desired estimate.

Proposition 2.2 Let $m>1$ and $\mathbf{u}$ be a local weak sub(super)-solution to (1.1)-(1.2) in $E_{T}$. There exists a constant $\gamma>0$, such that for all cylinders $Q_{R, S}:=K_{R}\left(x_{o}\right) \times\left(t_{o}-S, t_{o}\right] \subset E_{T}$, 
every $k \in \mathbb{R}$, every $i \in\{1, \cdots, n\}$, and every non-negative, piecewise smooth cutoff function $\zeta$ in $Q_{R, S}$ vanishing on $\partial K_{R}\left(x_{o}\right)$,

$$
\begin{aligned}
& \underset{t_{o}-S<t<t_{o}}{\operatorname{ess} \sup } \int_{K_{R}\left(x_{o}\right)}\left(u_{i}-k\right)_{ \pm}^{2} \zeta^{2}(x, t) \mathrm{d} x+\iint_{Q_{R, S}}|\mathbf{u}|^{m-1}\left|D\left(u_{i}-k\right)_{ \pm}\right|^{2} \zeta^{2} \mathrm{~d} x \mathrm{~d} t \\
& \leq \gamma \iint_{Q_{R, S}}|\mathbf{u}|^{m-1}\left(u_{i}-k\right)_{ \pm}^{2}|D \zeta|^{2} \mathrm{~d} x \mathrm{~d} t+\gamma \iint_{Q_{R, S}}\left(u_{i}-k\right)_{ \pm}^{2}\left|\zeta_{t}\right| \mathrm{d} x \mathrm{~d} t \\
& +\int_{K_{R}\left(x_{o}\right)}\left(u_{i}-k\right)_{ \pm}^{2} \zeta^{2}\left(x, t_{o}-S\right) \mathrm{d} x .
\end{aligned}
$$

Proof In the weak formulation of the sub(super)-solution to the $i$-th equation of the system $(1.1)-(1.2)$ we take the test function $\pm\left(u_{i}-k\right)_{ \pm} \psi_{\varepsilon} \zeta^{2}$ over the cylinder $K_{\varrho}\left(x_{o}\right) \times\left(t_{o}-\right.$ $\left.S, t_{o}\right]$, where $\psi_{\varepsilon}$ is defined in the proof of Proposition 2.1. Then a time mollification as in Proposition 2.1 will justify the use of the test function. We refrain from further elaboration and refer the reader to [6, Chapter 3, Proposition 6.1] for analogous calculations.

Remark 2.1 The energy estimate in Proposition 2.2 does not hold when $0<m<1$ in general, since the integral containing $|\mathbf{u}|^{m-1}$ might diverge. Nevertheless if we restrict ourselves to $k>0$ for sub-solutions with positive truncations, or $k<0$ for super-solutions with negative truncations, then the above estimate still holds for locally bounded solutions, when $0<m<1$. Indeed, one observes that the notion of solution and $\mathbf{u} \in L_{\mathrm{loc}}^{\infty}\left(E_{T}\right)$ implies that $D \mathbf{u} \in L_{\text {loc }}^{2}\left(E_{T}\right)$, when $0<m<1$.

\section{De Giorgi-type Lemmas}

Suppose $\left(x_{o}, t_{o}\right)+Q_{\varrho}(\theta) \subset E_{T}$, and for a locally bounded function $\mathbf{u}$ in $E_{T}$, we introduce a quantity $M$ satisfying

$$
2 M \geq \max _{1 \leq i \leq n} \operatorname{ess}_{\left(x_{o}, t_{o}\right)+Q_{\varrho}(\theta)}\left|u_{i}\right| .
$$

First of all, we have the following De Giorgi type lemma for the general system (1.1) - (1.2).

Lemma 3.1 Let $\mathbf{u}$ be a locally bounded, local weak sub(super)-solution to (1.1) - (1.2) in $E_{T}$. There exists a positive constant $v \in(0,1)$ depending only on $M, \theta$ and the data, such that if for some $i \in\{1, \cdots, n\}$,

$$
\left|\left[\mp u_{i} \leq M\right] \cap\left[\left(x_{o}, t_{o}\right)+Q_{\varrho}(\theta)\right]\right| \leq v\left|Q_{\varrho}(\theta)\right|,
$$

then

$$
\mp u_{i} \geq \frac{1}{2} M \text { a.e. in }\left(x_{o}, t_{o}\right)+Q_{\frac{\varrho}{2}}(\theta) \text {. }
$$

Moreover,

$$
v=\frac{\gamma M^{1-m} / \theta}{\left(1+M^{1-m} / \theta\right)^{\frac{N+2}{2}}}
$$

for some $\gamma>0$ depending only on the data.

Remark 3.1 If we set $\theta=M^{1-m}$ in Lemma 3.1, then $v$ becomes a positive constant depending only on the data. Moreover, Lemma 3.1 can be stated for cylinders of the type $\mathcal{Q}_{\varrho}(\vartheta)$, with the choice $\vartheta=M^{\frac{m-1}{2}}$. 


\subsection{Proof of Lemma 3.1 when $m>1$}

Assume $\left(x_{o}, t_{o}\right)=(0,0)$. We will present the proof for super-solutions only, sub-solutions being similar. In order to employ the energy estimate in Proposition 2.2, we set

$$
k_{n}=\frac{M}{2}+\frac{M}{2^{n+1}}, \quad \varrho_{n}=\varrho+\frac{\varrho}{2^{n}}, \quad K_{n}=K_{\varrho_{n}}, \quad Q_{n}=K_{n} \times\left(-\theta \varrho_{n}^{2}, 0\right] .
$$

Introduce the test function $\zeta$ vanishing on the parabolic boundary of $Q_{n}$ and equal to identity in $Q_{n+1}$, such that

$$
|D \zeta| \leq \gamma \frac{2^{n}}{\varrho} \quad \text { and } \quad\left|\zeta_{t}\right| \leq \gamma \frac{4^{n}}{\theta \varrho^{2}}
$$

In this setting, the energy estimate may be written as

$$
\begin{aligned}
& \underset{-\theta \varrho_{n}^{2}<t<0}{\operatorname{ess} \sup } \int_{K_{n}}\left(u_{i}-k_{n}\right)_{-}^{2} \zeta^{2}(x, t) \mathrm{d} x+\iint_{Q_{n}}|\mathbf{u}|^{m-1}\left|D\left[\left(u_{i}-k_{n}\right)_{-} \zeta\right]\right|^{2} \mathrm{~d} x \mathrm{~d} t \\
& \quad \leq \gamma \iint_{Q_{n}}|\mathbf{u}|^{m-1}\left(u_{i}-k_{n}\right)_{-}^{2}|D \zeta|^{2} \mathrm{~d} x \mathrm{~d} t+\gamma \iint_{Q_{n}}\left(u_{i}-k_{n}\right)_{-}^{2}\left|\zeta_{t}\right| \mathrm{d} x \mathrm{~d} t \\
& \quad \leq \gamma\left(\frac{4^{n}}{\varrho^{2}} M^{m+1}+\frac{4^{n}}{\theta \varrho^{2}} M^{2}\right)\left|A_{n}\right|,
\end{aligned}
$$

where we have set $A_{n}:=\left[u_{i}<k_{n}\right] \cap Q_{n}$. To estimate the left-hand side from below we introduce

$$
\tilde{u}_{i}:=\max \left\{u_{i}, \frac{1}{4} M\right\} .
$$

Then for the time part we estimate

$$
\int_{K_{n}}\left(u_{i}-k_{n}\right)_{-}^{2} \zeta^{2}(x, t) \mathrm{d} x \geq \int_{K_{n}}\left(\tilde{u}_{i}-k_{n}\right)_{-}^{2} \zeta^{2}(x, t) \mathrm{d} x .
$$

For the space part, we first observe that

$$
\begin{aligned}
& \left(\frac{1}{4} M\right)^{m-1} \iint_{Q_{n}}\left|D\left[\left(\tilde{u}_{i}-k_{n}\right)_{-} \zeta\right]\right|^{2} \mathrm{~d} x \mathrm{~d} t \\
& \leq \iint_{Q_{n}}\left|\tilde{u}_{i}\right|^{m-1}\left|D\left[\left(\tilde{u}_{i}-k_{n}\right)_{-} \zeta\right]\right|^{2} \mathrm{~d} x \mathrm{~d} t \\
& =\iint_{Q_{n} \cap\left[\tilde{u}_{i}=u_{i}\right]}\left|\tilde{u}_{i}\right|^{m-1}\left|D\left[\left(\tilde{u}_{i}-k_{n}\right)_{-} \zeta\right]\right|^{2} \mathrm{~d} x \mathrm{~d} t \\
& \quad+\iint_{Q_{n} \cap\left[u_{i}<\tilde{u}_{i}\right]}\left|\tilde{u}_{i}\right|^{m-1}\left|D\left[\left(\tilde{u}_{i}-k_{n}\right)_{-} \zeta\right]\right|^{2} \mathrm{~d} x \mathrm{~d} t \\
& \quad \leq \iint_{Q_{n}}|\mathbf{u}|^{m-1}\left|D\left[\left(u_{i}-k_{n}\right)_{-} \zeta\right]\right|^{2} \mathrm{~d} x \mathrm{~d} t+\gamma \frac{4^{n}}{Q^{2}} M^{m+1}\left|A_{n}\right| .
\end{aligned}
$$

Here in the last line we have used the fact that $\left[u_{i}<k_{n}\right]$ and $\left[\tilde{u}_{i}<k_{n}\right]$ coincide.

Collecting all the estimates above gives us that

$$
\begin{aligned}
& \underset{-\theta \varrho_{n}^{2}<t<0}{\operatorname{ess} \sup } \int_{K_{n}}\left(\tilde{u}_{i}-k_{n}\right)_{-}^{2} \zeta^{2}(x, t) \mathrm{d} x+M^{m-1} \iint_{Q_{n}}\left|D\left[\left(\tilde{u}_{i}-k_{n}\right)_{-} \zeta\right]\right|^{2} \mathrm{~d} x \mathrm{~d} t \\
& \quad \leq \gamma\left(\frac{4^{n}}{\varrho^{2}} M^{m+1}+\frac{4^{n}}{\theta \varrho^{2}} M^{2}\right)\left|A_{n}\right| .
\end{aligned}
$$


An application of Hölder's inequality and Sobolev's imbedding [4, Chapter I, Proposition 3.1] gives that

$$
\begin{aligned}
\left(\frac{M}{2^{n+2}}\right)^{2}\left|A_{n+1}\right| & \\
\leq & \iint_{Q_{n}}\left(\tilde{u}_{i}-k_{n}\right)_{-}^{2} \zeta^{2} \mathrm{~d} x \mathrm{~d} t \\
\leq & \left(\iint_{Q_{n}}\left[\left(\tilde{u}_{i}-k_{n}\right)_{-} \zeta\right]^{2 \frac{N+2}{N}} \mathrm{~d} x \mathrm{~d} t\right)^{\frac{N}{N+2}}\left|A_{n}\right|^{\frac{2}{N+2}} \\
\leq & \gamma\left(\iint_{Q_{n}}\left|D\left[\left(\tilde{u}_{i}-k_{n}\right)_{-} \zeta\right]\right|^{2} \mathrm{~d} x \mathrm{~d} t\right)^{\frac{N}{N+2}} \\
& \times\left(\operatorname{ess~sup~}_{-\theta \varrho_{n}^{2}<t<0} \int_{K_{n}}\left(\tilde{u}_{i}-k_{n}\right)_{-}^{2} \zeta^{2}(x, t) \mathrm{d} x\right)^{\frac{2}{N+2}}\left|A_{n}\right|^{\frac{2}{N+2}} \\
\leq & \gamma M^{\frac{(1-m) N}{N+2}}\left(\frac{4^{n}}{\varrho^{2}} M^{1+m}+\frac{4^{n}}{\theta \varrho^{2}} M^{2}\right)\left|A_{n}\right|^{1+\frac{2}{N+2}} .
\end{aligned}
$$

In terms of $Y_{n}=\left|A_{n}\right| /\left|Q_{n}\right|$, this can be rewritten as

$$
Y_{n+1} \leq \gamma 2^{4 n}\left(\frac{\theta}{M^{1-m}}\right)^{\frac{2}{N+2}}\left(1+\frac{M^{1-m}}{\theta}\right) Y_{n}^{1+\frac{2}{N+2}} .
$$

Hence by [4, Chapter I, Lemma 4.1] $Y_{n} \rightarrow 0$, if we require that

$$
Y_{o} \leq \gamma \frac{M^{1-m}}{\theta}\left[1+\frac{M^{1-m}}{\theta}\right]^{-\frac{N+2}{2}}=: v \text {. }
$$

The proof is concluded.

\subsection{Proof of Lemma 3.1 when $0<m<1$}

Assume $\left(x_{o}, t_{o}\right)=(0,0)$. In order to employ the energy estimate in Proposition 2.1, we notice first that, for $\tilde{k}<k$ we have

$$
\int_{u_{i}}^{k}(\varphi(s)-\varphi(k))_{-} \mathrm{d} s \geq \int_{u_{i}}^{\tilde{k}}(\varphi(s)-\varphi(k))_{-} \mathrm{d} s \geq(\varphi(k)-\varphi(\tilde{k}))\left(u_{i}-\tilde{k}\right)_{-} .
$$

As a result, the energy estimate reads

$$
\begin{aligned}
& (\varphi(k)-\varphi(\tilde{k})) \underset{-\theta \varrho^{2}<t<0}{\operatorname{ess} \sup _{K_{\varrho}}} \int_{K}\left(u_{i}-\tilde{k}\right)_{-} \zeta^{2} \mathrm{~d} x+\iint_{Q_{\varrho}(\theta)}|\mathbf{u}|^{2(m-1)}\left|D\left(u_{i}-\tilde{k}\right)_{-}\right|^{2} \zeta^{2} \mathrm{~d} x \mathrm{~d} t \\
& \quad \leq \gamma \iint_{Q_{\varrho}(\theta)}\left(u_{i}-k\right)_{-}^{2 m}|D \zeta|^{2} \mathrm{~d} x \mathrm{~d} t+\gamma \iint_{Q_{\varrho}(\theta)}\left(u_{i}-k\right)_{-}^{1+m}\left|\zeta_{t}\right| \mathrm{d} x \mathrm{~d} t .
\end{aligned}
$$

In order to use this energy estimate, we set for $n=0,1, \cdots$,

$$
\begin{aligned}
& k_{n}=\frac{M}{2}+\frac{M}{2^{n+1}}, \quad \tilde{k}_{n}=\frac{k_{n}+k_{n+1}}{2}, \quad \varrho_{n}=\varrho+\frac{\varrho}{2^{n}}, \quad \tilde{\varrho}_{n}=\frac{\varrho_{n}+\varrho_{n+1}}{2}, \\
& K_{n}=K_{\varrho_{n}}, \quad K_{\tilde{\varrho}_{n}}, \quad Q_{n}=K_{n} \times\left(-\theta \varrho_{n}^{2}, 0\right], \quad \widetilde{Q}_{n}=\widetilde{K}_{n} \times\left(-\theta \tilde{\varrho}_{n}^{2}, 0\right] .
\end{aligned}
$$


Introduce the test function $\zeta$ vanishing on the parabolic boundary of $Q_{n}$ and equal to identity in $\widetilde{Q}_{n}$, such that

$$
|D \zeta| \leq \gamma \frac{2^{n}}{\varrho} \quad \text { and } \quad\left|\zeta_{t}\right| \leq \gamma \frac{4^{n}}{\theta \varrho^{2}}
$$

In this setting, the energy estimate may be written as

$$
\begin{aligned}
& \left(\varphi\left(k_{n}\right)-\varphi\left(\tilde{k}_{n}\right)\right) \underset{-\theta \tilde{\varrho}_{n}^{2}<t<0}{\operatorname{ess} \sup } \int_{\widetilde{K}_{n}}\left(u_{i}-\tilde{k}_{n}\right)_{-} \mathrm{d} x+\iint_{\widetilde{Q}_{n}}|\mathbf{u}|^{2(m-1)}\left|D\left(u_{i}-\tilde{k}_{n}\right)_{-}\right|^{2} \mathrm{~d} x \mathrm{~d} t \\
& \quad \leq \gamma \iint_{Q_{n}}\left(u_{i}-k_{n}\right)_{-}^{2 m}|D \zeta|^{2} \mathrm{~d} x \mathrm{~d} t+\gamma \iint_{Q_{n}}\left(u_{i}-k_{n}\right)_{-}^{1+m}\left|\zeta_{t}\right| \mathrm{d} x \mathrm{~d} t \\
& \quad \leq \gamma\left(\frac{4^{n}}{\varrho^{2}} M^{2 m}+\frac{4^{n}}{\theta \varrho^{2}} M^{1+m}\right)\left|A_{n}\right|,
\end{aligned}
$$

where $A_{n}=\left[u_{i}<k_{n}\right] \cap Q_{n}$.

Noting that $k_{n} \leq M$ and $\varphi^{\prime}(s)=m|s|^{m-1}$, we may estimate the terms on the left-hand side of the energy estimate from below by

$$
\begin{aligned}
& \varphi\left(k_{n}\right)-\varphi\left(\tilde{k}_{n}\right)=\int_{\tilde{k}_{n}}^{k_{n}} \varphi^{\prime}(s) \mathrm{d} s \geq m M^{m-1}\left(k_{n}-\tilde{k}_{n}\right)=\frac{m M^{m}}{2^{n+3}}, \\
& \iint_{\widetilde{Q}_{n}}|\mathbf{u}|^{2(m-1)}\left|D\left(u_{i}-\tilde{k}_{n}\right)_{-}\right|^{2} \mathrm{~d} x \mathrm{~d} t \geq\left(\gamma_{o} M\right)^{2(m-1)} \iint_{\widetilde{Q}_{n}}\left|D\left(u_{i}-\tilde{k}_{n}\right)_{-}\right|^{2} \mathrm{~d} x \mathrm{~d} t .
\end{aligned}
$$

In the last line we have used (3.1), and consequently $|\mathbf{u}| \leq 2 n^{\frac{1}{2}} M$, where $n$ is the number of equations in (I). In order not to confuse it with the integral index $n$, we have used $\gamma_{o}$ to denote $2 n^{\frac{1}{2}}$.

Collecting all the estimates above gives us that

$$
\begin{aligned}
& \frac{M^{m}}{2^{n}} \underset{-\theta \tilde{Q}_{n}^{2}<t<0}{\operatorname{essup}} \int_{\widetilde{K}_{n}}\left(u_{i}-\tilde{k}_{n}\right)_{-} \mathrm{d} x+M^{2(m-1)} \iint_{\widetilde{Q}_{n}}\left|D\left(u_{i}-\tilde{k}_{n}\right)_{-}\right|^{2} \mathrm{~d} x \mathrm{~d} t \\
& \quad \leq \gamma\left(\frac{4^{n}}{\varrho^{2}} M^{2 m}+\frac{4^{n}}{\theta \varrho^{2}} M^{1+m}\right)\left|A_{n}\right| .
\end{aligned}
$$

Now setting $\phi$ to be a cutoff function which vanishes on the parabolic boundary of $\widetilde{Q}_{n}$ and equals identity in $Q_{n+1}$, an application of the Sobolev imbedding [4, Chapter I, Proposition 3.1] gives that

$$
\begin{aligned}
& \left(\frac{M}{2^{n+3}}\right)^{2}\left|A_{n+1}\right| \leq \iint_{\widetilde{Q}_{n}}\left(u_{i}-\tilde{k}_{n}\right)_{-}^{2} \phi^{2} \mathrm{~d} x \mathrm{~d} t \\
& \leq\left(\iint_{\widetilde{Q}_{n}}\left[\left(u_{i}-\tilde{k}_{n}\right)_{-} \phi\right]^{2 \frac{N+1}{N}} \mathrm{~d} x \mathrm{~d} t\right)^{\frac{N}{N+1}}\left|A_{n}\right|^{\frac{1}{N+1}} \\
& \leq \gamma\left(\iint_{\widetilde{Q}_{n}}\left|D\left(u_{i}-\tilde{k}_{n}\right)_{-}\right|^{2} \mathrm{~d} x \mathrm{~d} t\right)^{\frac{N}{N+1}} \\
& \quad \times\left(\operatorname{esssup}_{-\theta \tilde{\varrho}_{n}^{2}<t<0} \int_{\widetilde{K}_{n}}\left(u_{i}-\tilde{k}_{n}\right)_{-} \mathrm{d} x\right)^{\frac{2}{N+1}}\left|A_{n}\right|^{\frac{1}{N+1}} \\
& \leq \gamma\left(\frac{M^{m}}{2^{n}}\right)^{-\frac{2}{N+1}} M^{\frac{2(1-m) N}{N+1}}\left(\frac{4^{n}}{\varrho^{2}} M^{2 m}+\frac{4^{n}}{\theta \varrho^{2}} M^{1+m}\right)^{\frac{N+2}{N+1}}\left|A_{n}\right|^{1+\frac{2}{N+1}} .
\end{aligned}
$$


In terms of $Y_{n}=\left|A_{n}\right| /\left|Q_{n}\right|$, this can be rewritten as

$$
Y_{n+1} \leq \gamma b^{n}\left(\frac{\theta}{M^{1-m}}\right)^{\frac{2}{N+1}}\left(1+\frac{M^{1-m}}{\theta}\right)^{\frac{N+2}{N+1}} Y_{n}^{1+\frac{2}{N+1}}
$$

where $b$ and $\gamma$ depend only on the data. Hence by [4, Chapter I, Lemma 4.1], $Y_{n} \rightarrow 0$ if we require that

$$
Y_{o} \leq \gamma \frac{M^{1-m}}{\theta}\left[1+\frac{M^{1-m}}{\theta}\right]^{-\frac{N+2}{2}}=: v .
$$

The proof is concluded.

\section{Reduction of the supreme of solutions}

Let $M$ be defined in (3.1) and $v$ be the constant determined in Lemma 3.1 by taking $\theta=$ $M^{1-m}$. Suppose for all $i \in\{1, \cdots, n\}$, the following measure theoretical information holds

$$
\left\{\begin{array}{c}
\left|\left[u_{i} \leq M\right] \cap\left[\left(x_{o}, t_{o}\right)+Q_{\varrho}(\theta)\right]\right|>v\left|Q_{\varrho}(\theta)\right|, \\
\left|\left[u_{i} \geq-M\right] \cap\left[\left(x_{o}, t_{o}\right)+Q_{\varrho}(\theta)\right]\right|>v\left|Q_{\varrho}(\theta)\right| .
\end{array}\right.
$$

We claim that if $(4.1)_{1}$ holds, then there exists $\eta \in(0,1)$ depending only on the data, such that

$$
u_{i} \leq 2 M(1-\eta) \quad \text { a.e. in }\left(x_{o}, t_{o}\right)+Q_{\frac{\rho}{2}}(\nu \theta) .
$$

Similarly, if $(4.1)_{2}$ holds, then there exists $\eta \in(0,1)$ depending only on the data, such that

$$
u_{i} \geq-2 M(1-\eta) \quad \text { a.e. in }\left(x_{o}, t_{o}\right)+Q_{\frac{\rho}{2}}(\nu \theta) .
$$

Combining the two estimates, the supreme of $|\mathbf{u}|$ is reduced in a smaller cylinder, i.e.

$$
\max _{1 \leq i \leq n} \underset{\left(x_{o}, t_{o}\right)+Q_{\frac{\rho}{2}}(\nu \theta)}{\operatorname{ess} \sup }\left|u_{i}\right| \leq 2 M(1-\eta) .
$$

Remark 4.1 The above statement can be made for cylinders of the type $\mathcal{Q}_{\varrho}(\vartheta)$ with $\vartheta=$ $M^{\frac{m-1}{2}}$. Namely, omitting the reference to $\left(x_{o}, t_{o}\right)$, the following implications hold:

$$
\begin{gathered}
\left|\left[u_{i} \leq M\right] \cap \mathcal{Q}_{\varrho}(\vartheta)\right|>v\left|\mathcal{Q}_{\varrho}(\vartheta)\right| \Longrightarrow u_{i} \leq 2 M(1-\eta) \quad \text { a.e. in } \mathcal{Q}_{\frac{\varrho}{2}}\left(v^{-\frac{1}{2}} \vartheta\right) \\
\left|\left[u_{i} \geq-M\right] \cap \mathcal{Q}_{\varrho}(\vartheta)\right|>v\left|\mathcal{Q}_{\varrho}(\vartheta)\right| \Longrightarrow u_{i} \geq-2 M(1-\eta) \quad \text { a.e. in } \mathcal{Q}_{\frac{\varrho}{2}}\left(v^{-\frac{1}{2}} \vartheta\right)
\end{gathered}
$$

Let us treat $(4.1)_{1}$ for instance. For simplicity, we assume $\left(x_{o}, t_{o}\right)=(0,0)$. Recalling the definition of $M$ in (3.1), we always have $|\mathbf{u}| \leq 2 n^{\frac{1}{2}} M$ in $Q_{\varrho}(\theta)$. We will work with $\mathbf{u}$ as a sub-solution within the smaller set

$$
\left[M \leq|\mathbf{u}| \leq 2 n^{\frac{1}{2}} M\right] \cap Q_{\varrho}(\theta) .
$$

This is realized by working with the energy estimate in Proposition 2.2 about $\left(u_{i}-k\right)_{+}$ for some $k \geq M$. As we have already mentioned in Remark 2.1, the energy estimate in Proposition 2.2 holds for all $m>0$ here, since the level $k>0$. This energy estimate is the basis for the following Lemmas $4.1-4.3$. 
To start with, we observe that there exists some $s \in\left[-\theta \varrho^{2},-\frac{1}{2} v \theta \varrho^{2}\right]$, such that

$$
\left|\left[u_{i}(\cdot, s) \leq M\right] \cap K_{\varrho}\right|>\frac{1}{2} v\left|K_{\varrho}\right| \cdot
$$

Indeed, if the above inequality were not to hold for any $s$ in the given interval, then we would have arrived at a contradiction to $(4.1)_{1}$ :

$$
\begin{aligned}
\left|\left[u_{i} \leq M\right] \cap Q_{\varrho}(\theta)\right|= & \int_{-\theta \varrho^{2}}^{-\frac{1}{2} \nu \theta \varrho^{2}}\left|\left[u_{i}(\cdot, s) \leq M\right] \cap K_{\varrho}\right| \mathrm{d} s \\
& +\int_{-\frac{1}{2} \nu \theta \varrho^{2}}^{0}\left|\left[u_{i}(\cdot, s) \leq M\right] \cap K_{\varrho}\right| \mathrm{d} s \\
\leq & v\left|Q_{\varrho}(\theta)\right| .
\end{aligned}
$$

\subsection{Propagation of measure information}

Now we introduce a lemma concerning the time propagation of measure theoretical information in (4.4).

Lemma 4.1 Assume (4.4) holds. There exist $\delta$ and $\varepsilon$ in $(0,1)$, depending only on the data and $v$, such that

$$
\left|\left[u_{i}(\cdot, s) \leq 2 M-\varepsilon M\right] \cap K_{\varrho}(y)\right| \geq \frac{1}{4} v\left|K_{\varrho}(y)\right| \quad \text { for all } t \in\left(s, s+\delta M^{1-m} \varrho^{2}\right] .
$$

Proof Use the energy estimate in Proposition 2.2 for sub-solutions in the cylinder $Q=$ $K_{\varrho} \times\left(0, \delta M^{1-m} \varrho^{2}\right]$, with $k=M$ and choose a standard non-negative cutoff function $\zeta(x)$ that equals 1 on $K_{(1-\sigma) \varrho}$ and vanishes on $\partial K_{\varrho}$ satisfying $|D \zeta| \leq(\sigma \varrho)^{-1}$; in such a case, we have for all $0<t<\delta M^{1-m} \varrho^{2}$,

$$
\begin{aligned}
\int_{K_{\varrho}}\left(u_{i}-k\right)_{+}^{2} \zeta^{2}(x, t) \mathrm{d} x \leq & \int_{K_{\varrho}}\left(u_{i}-k\right)_{+}^{2} \zeta^{2}(x, 0) \mathrm{d} x \\
& +\gamma \iint_{Q}|\mathbf{u}|^{m-1}\left(u_{i}-k\right)_{+}^{2}|D \zeta|^{2} \mathrm{~d} x \mathrm{~d} t \\
& \leq \int_{K_{\varrho}}\left(u_{i}-k\right)_{+}^{2} \zeta^{2}(x, 0) \mathrm{d} x+\gamma\left|K_{\varrho}\right| \frac{\delta M^{2}}{\sigma^{2}} \\
& \leq M^{2}\left[\left(1-\frac{1}{2} v\right)+\gamma \frac{\delta}{\sigma^{2}}\right]\left|K_{\varrho}\right| .
\end{aligned}
$$

In estimating the term containing $|\mathbf{u}|^{m-1}$ we have evoked (4.3).

The left-hand side of the energy estimate can be bounded from below by

$$
\begin{aligned}
\int_{K_{\varrho}}\left(u_{i}-k\right)_{+}^{2} \zeta^{2}(x, t) \mathrm{d} x & \geq \int_{K_{(1-\sigma) \varrho} \cap\left[u_{i} \geq 2 M-\varepsilon M\right]}\left(u_{i}-k\right)_{+}^{2} \zeta^{2}(x, t) \mathrm{d} x \\
& \geq(1-\varepsilon)^{2} M^{2}\left|A_{\ell,(1-\sigma) \varrho}(t)\right|
\end{aligned}
$$

where we have defined

$$
A_{\ell,(1-\sigma) \varrho}(t)=\left[u_{i}(\cdot, t) \geq \ell\right] \cap K_{(1-\sigma) \varrho} \quad \text { and } \quad \ell=2 M-\varepsilon M .
$$

Notice that

$$
\left|A_{\ell, \varrho}(t)\right|=\left|A_{\ell,(1-\sigma) \varrho}(t) \cup\left(A_{\ell, \varrho}(t)-A_{\ell,(1-\sigma) \varrho}(t)\right)\right|
$$




$$
\begin{aligned}
& \leq\left|A_{\ell,(1-\sigma) \varrho}(t)\right|+\left|K_{\varrho}-K_{(1-\sigma) \varrho}\right| \\
& \leq\left|A_{\ell,(1-\sigma) \varrho}(t)\right|+N \sigma\left|K_{\varrho}\right| .
\end{aligned}
$$

Collecting all the above estimates yields that

$$
\left|A_{\ell, \varrho}(t)\right| \leq \frac{1}{(1-\varepsilon)^{2}}\left[\left(1-\frac{1}{2} \nu\right)+\gamma \frac{\delta}{\sigma^{2}}\right]\left|K_{\varrho}\right|+N \sigma\left|K_{\varrho}\right| .
$$

Finally, this allows us to choose the various parameters quantitatively. Indeed, we may assume $\varepsilon$ is so small that $(1-\varepsilon)^{2} \geq \frac{1}{2}$ and choose

$$
\sigma=\frac{v}{16 N}, \quad \gamma \frac{\delta}{\sigma^{2}} \leq \frac{v}{32} .
$$

Then the above estimate is simplified as

$$
\left|A_{\ell, \varrho}(t)\right| \leq\left[\frac{1-\frac{1}{2} \nu}{(1-\varepsilon)^{2}}+\frac{v}{8}\right]\left|K_{\varrho}\right| .
$$

To conclude, we may choose $\varepsilon$ such that

$$
\frac{1-\frac{1}{2} v}{(1-\varepsilon)^{2}}+\frac{v}{8}<1-\frac{v}{4}, \quad \text { i.e. } \varepsilon \approx \frac{v}{8}
$$

This completes the proof.

\subsection{A shrinking lemma}

Based on the measure theoretical information obtained in Lemma 4.1 for each slice $K_{\varrho} \times\{t\}$, where $t \in\left(s, s+\delta \theta \varrho^{2}\right]$, we may prove the following shrinking lemma.

Lemma 4.2 Assume (4.4) holds. There exists $\gamma>0$ depending only on the data, such that for any positive integer $j_{*}$, we have

$$
\left|\left[u_{i} \geq 2 M-\frac{\varepsilon M}{2^{j_{*}}}\right] \cap Q\right| \leq \frac{\gamma}{v \sqrt{\delta j_{*}}}|Q|, \quad \text { where } Q=K_{\varrho} \times\left(s, s+\delta \theta \varrho^{2}\right] .
$$

Proof We employ the energy estimate of Proposition 2.2 in a larger cylinder $Q^{\prime}=K_{2 \varrho} \times$ $\left(s, s+\delta \theta \varrho^{2}\right]$, with levels $k_{j}=2 M\left(1-\frac{\varepsilon}{2^{j+1}}\right)$ for $j \geq 0$ and a cutoff function $\zeta$ in $K_{2 \varrho}$ that is equal to 1 in $K_{\varrho}$ and vanishes on $\partial K_{2 \varrho}$, such that $|D \zeta| \leq 2 \varrho^{-1}$. Then, we obtain

$$
\begin{aligned}
\iint_{Q}|\mathbf{u}|^{m-1}\left|D\left(u_{i}-k_{j}\right)_{+}\right|^{2} \mathrm{~d} x \mathrm{~d} t \leq & \gamma \int_{K_{2 \varrho}}\left(u_{i}-k_{j}\right)_{+}^{2}(x, s) \mathrm{d} x \\
& +\gamma \iint_{Q^{\prime}}|\mathbf{u}|^{m-1}\left(u_{i}-k_{j}\right)_{+}^{2}|D \zeta|^{2} \mathrm{~d} x \mathrm{~d} t .
\end{aligned}
$$

Recalling (3.1) and observing that on the set $\left[u_{i}>k_{j}\right]$, there holds

$$
M<u_{i} \leq|\mathbf{u}| \leq 2 n^{\frac{1}{2}} M .
$$

Hence the above energy estimate yields

$$
\iint_{Q}\left|D\left(u_{i}-k_{j}\right)_{+}\right|^{2} \mathrm{~d} x \mathrm{~d} t \leq \frac{\gamma}{\delta \varrho^{2}}\left(\frac{\varepsilon M}{2^{j}}\right)^{2}|Q| .
$$


Next, we apply [4, Chapter I, Lemma 2.2] to $u_{i}(\cdot, t)$ for $t \in\left(s, s+\delta \theta \varrho^{2}\right]$ over the cube $K_{\varrho}$, for levels $k_{j}<k_{j+1}$. Taking into account the measure theoretical information from Lemma 4.1:

$$
\left|\left[u_{i}(\cdot, t) \leq 2 M-\varepsilon M\right] \cap K_{\varrho}\right| \geq \frac{1}{4} v\left|K_{\varrho}\right| \quad \text { for all } t \in\left(s, s+\delta \theta \varrho^{2}\right],
$$

together with an application of Hölder's inequality, this gives

$$
\begin{aligned}
& \frac{\varepsilon M}{2^{j+1}}\left|\left[u_{i}(\cdot, t)>k_{j+1}\right] \cap K_{\varrho}\right| \\
& \leq \frac{\gamma \varrho^{N+1}}{\left|\left[u_{i}(\cdot, t)<k_{j}\right] \cap K_{\varrho}\right|} \int_{\left[k_{j}<u_{i}(\cdot, t)<k_{j+1}\right] \cap K_{\varrho}}\left|D u_{i}\right| \mathrm{d} x \\
& \quad \leq \frac{\gamma \varrho}{v}\left(\int_{\left[k_{j}<u_{i}(\cdot, t)<k_{j+1}\right] \cap K_{\varrho}}\left|D u_{i}\right|^{2} \mathrm{~d} x\right)^{\frac{1}{2}} \\
& \quad \times\left|\left(\left[u_{i}(\cdot, t)>k_{j}\right]-\left[u_{i}(\cdot, t)>k_{j+1}\right]\right) \cap K_{\varrho}\right|^{\frac{1}{2}} .
\end{aligned}
$$

Set $A_{j}=\left[u_{i}>k_{j}\right] \cap Q$ and integrate the above estimate in $\mathrm{d} t$ over $\left(s, s+\delta \theta \varrho^{2}\right]$; we obtain by using the energy estimate and the Fubini theorem that

$$
\begin{aligned}
\frac{\varepsilon M}{2^{j}}\left|A_{j+1}\right| & \leq \frac{\gamma \varrho}{v}\left(\iint_{Q}\left|D\left(u_{i}-k_{j}\right)_{+}\right|^{2} \mathrm{~d} x \mathrm{~d} t\right)^{\frac{1}{2}}\left(\left|A_{j}\right|-\left|A_{j+1}\right|\right)^{\frac{1}{2}} \\
& \leq \frac{\gamma}{v \sqrt{\delta}} \frac{\varepsilon M}{2^{j}}|Q|^{\frac{1}{2}}\left(\left|A_{j}\right|-\left|A_{j+1}\right|\right)^{\frac{1}{2}} .
\end{aligned}
$$

Now square both sides of the above inequality to obtain

$$
\left|A_{j+1}\right|^{2} \leq \frac{\gamma}{v^{2} \delta}|Q|\left(\left|A_{j}\right|-\left|A_{j+1}\right|\right) .
$$

Add these inequalities from 0 to $j_{*}-1$ to obtain

$$
j_{*}\left|A_{j_{*}}\right|^{2} \leq \sum_{j=0}^{j_{*}-1}\left|A_{j+1}\right|^{2} \leq \frac{\gamma}{v^{2} \delta}|Q|^{2} .
$$

From this we conclude

$$
\left|A_{j_{*}}\right| \leq \frac{\gamma}{v \sqrt{\delta j_{*}}}|Q| .
$$

This is exactly the desired conclusion.

\subsection{A De Giorgi-type lemma near the supreme}

Recall that $Q=K_{\varrho} \times\left(s, s+\delta \theta \varrho^{2}\right]$. We need the following De Giorgi-type lemma in the set $Q$. The proof is analogous to the one for Lemma 3.1.

Lemma 4.3 Let $\xi \in(0,1)$. There exists a positive constant $v_{o}$ depending only on the data and $\delta$, but independent of $\xi$, such that if

$$
\left|\left[u_{i} \geq 2 M-\xi M\right] \cap Q\right| \leq v_{o}|Q|,
$$

then

$$
u_{i} \leq 2 M-\frac{1}{2} \xi M \quad \text { a.e. in } K \frac{\varrho}{2} \times\left(s+\frac{3}{4} \delta \theta \varrho^{2}, s+\delta \theta \varrho^{2}\right]
$$


Proof For simplicity, we may fix the top of the cylinder $Q$, i.e. $s+\delta \theta \varrho^{2}$, at 0 . For $n=0,1, \ldots$ we set

$$
\begin{aligned}
& k_{n}=2 M-\frac{\xi M}{2}-\frac{\xi M}{2^{n+1}}, \quad \varrho_{n}=\frac{\varrho}{2}+\frac{\varrho}{2^{n+1}}, \\
& K_{n}=K_{\varrho_{n}}, \quad Q_{n}=K_{n} \times\left(-\delta \theta \varrho_{n}^{2}, 0\right] .
\end{aligned}
$$

Write down the energy estimate in Proposition 2.2 over the cylinder $Q_{n}$. Taking also into account the set $\left[u_{i}>k_{n}\right]$, we are working in the set (4.3), i.e. there holds

$$
M<u_{i} \leq|\mathbf{u}| \leq \gamma_{o} M .
$$

Here $\gamma_{o}=2 n^{\frac{1}{2}}$ and $n$ represents the number of equations in the system (1.1). We also use $n$ as a generic integral index in the proof here.

This gives the energy estimate

$$
\begin{aligned}
& \underset{-\delta \theta \varrho_{n}^{2}<t \leq 0}{\operatorname{ess} \sup } \int_{K_{n} \times\{t\}}\left(u_{i}-k_{n}\right)_{+}^{2} \zeta^{2} \mathrm{~d} x+M^{m-1} \iint_{Q_{n}}\left|D\left[\left(u_{i}-k_{n}\right)_{+} \zeta\right]\right|^{2} \mathrm{~d} x \mathrm{~d} t \\
& \leq \gamma 2^{2 n}(\xi M)^{2} \iint_{Q_{n}}\left(\frac{M^{m-1}}{\varrho^{2}}+\frac{1}{\delta \theta \varrho^{2}}\right) \chi_{\left[u_{i}>k_{n}\right]} \mathrm{d} x \mathrm{~d} t \\
& \leq \gamma \frac{2^{2 n}}{\varrho^{2}}(\xi M)^{2} M^{m-1}\left(1+\frac{M^{1-m}}{\delta \theta}\right)\left|A_{n}\right|
\end{aligned}
$$

where we have set $A_{n}:=\left[u_{i}>k_{n}\right] \cap Q_{n}$.

Applying Hölder's inequality and the Sobolev embedding [4, Chapter I, Proposition 3.1], and recalling that $\zeta=1$ on $Q_{n+1}$, we get

$$
\begin{aligned}
& \frac{(\xi M)^{2}}{2^{2(n+2)}}\left|A_{n+1}\right| \leq \iint_{Q_{n+1}}\left(u_{i}-k_{n}\right)_{+}^{2} \mathrm{~d} x \mathrm{~d} t \\
& \leq\left(\iint_{Q_{n}}\left[\left(u_{i}-k_{n}\right)_{+} \zeta\right]^{2 \frac{N+2}{N}} \mathrm{~d} x \mathrm{~d} t\right)^{\frac{N}{N+2}}\left|A_{n}\right|^{\frac{2}{N+2}} \\
& \leq \gamma\left(\iint_{Q_{n}}\left|D\left[\left(u_{i}-k_{n}\right)_{+} \zeta\right]\right| \mathrm{d} x \mathrm{~d} t\right)^{\frac{N}{N+2}} \\
& \quad \times\left(\operatorname{ess~sup}_{-\delta \theta \varrho_{n}^{2}<t \leq 0} \int_{K_{n} \times\{t\}}\left[\left(u_{i}-k_{n}\right)_{+} \zeta\right]^{2} \mathrm{~d} x\right)^{\frac{2}{N+2}}\left|A_{n}\right|^{\frac{2}{N+2}}
\end{aligned}
$$

for a constant $\gamma$ depending only upon $N$. Combine this with the energy estimate to get

$$
\left|A_{n+1}\right| \leq \gamma \frac{2^{4 n}}{\varrho^{2}} M^{\frac{2(m-1)}{N+2}}\left(1+\frac{M^{1-m}}{\delta \theta}\right)\left|A_{n}\right|^{1+\frac{2}{N+2}} .
$$

In terms of $Y_{n}=\left|A_{n}\right| /\left|Q_{n}\right|$ this can be rewritten as

$$
Y_{n+1} \leq \gamma 2^{4 n}\left(\frac{\delta \theta}{M^{1-m}}\right)^{\frac{2}{N+2}}\left(1+\frac{M^{1-m}}{\delta \theta}\right) Y_{n}^{1+\frac{2}{N+2}} .
$$

By [4, Chapter I, Lemma 4.1], $Y_{n} \rightarrow 0$ as $n \rightarrow \infty$, provided

$$
Y_{o}=\frac{\left|A_{o}\right|}{\left|Q_{o}\right|} \leq \frac{1}{\gamma} \frac{M^{1-m} /(\delta \theta)}{\left(1+M^{1-m} /(\delta \theta)\right)^{\frac{N+2}{2}}}=: v_{o} .
$$

This finishes the proof. 


\subsection{Proof of (4.2)}

Now we are in a position to prove (4.2). The measure theoretical information $(4.1)_{1}$ gives (4.4) at some $s$ in $\left[-\theta \varrho^{2},-\frac{1}{2} \nu \theta \varrho^{2}\right]$. Whereas (4.4) can be written in $K_{2 \varrho}$, i.e.

$$
\left|\left[u_{i}(\cdot, s) \leq M\right] \cap K_{2 \varrho}\right|>\frac{1}{2} \frac{1}{4^{N}} \nu\left|K_{2 \varrho}\right| .
$$

Next we may apply Lemma 4.1 with $\varrho$ and $v$ replaced by $2 \varrho$ and $\frac{1}{4^{N}} v$ respectively. With $\delta$ and $\varepsilon$ fixed in terms of these parameters as in Lemma 4.1, we may fix $v_{o}$ in Lemma 4.3 depending on the data and also on $\delta$. Then we may choose $\xi=\varepsilon 2^{-j_{*}}$, whereas $j_{*}$ is chosen according to Lemma 4.2 , such that

$$
\frac{\gamma 4^{N}}{v \sqrt{\delta j_{*}}} \leq v_{o}
$$

Thus Lemma 4.3 yields that

$$
u_{i} \leq 2 M-\frac{\varepsilon M}{2^{j_{*}+1}} \quad \text { a.e. in } K_{\varrho} \times\left(s+\frac{3}{4} \delta \theta(2 \varrho)^{2}, s+\delta \theta(2 \varrho)^{2}\right] .
$$

If $s+\delta \theta(2 \varrho)^{2} \geq 0$, we are done. If not, we may run the machinery of Lemmas $4.1-4.3$ again, keeping in mind that we are always in the set (4.3) where degeneracy or singularity is avoided. In fact, the quantitative information in (4.5) gives a similar condition as (4.4), with $\frac{1}{2} v=1$. Then reasoning as above using Lemmas $4.1-4.3$ would yield $\bar{\delta}$ and $\bar{\xi}$ depending only on the data such that,

$$
u_{i} \leq 2 M-\bar{\xi} \xi M \quad \text { a.e. in } K_{\varrho} \times\left(s+\frac{3}{4} \delta \theta(2 \varrho)^{2}, s+(\delta+\bar{\delta}) \theta(2 \varrho)^{2}\right] .
$$

After $L$ steps, we arrive at

$$
u_{i} \leq 2 M-\bar{\xi}^{L} \xi M \quad \text { a.e. in } K_{\varrho} \times\left(s+\frac{3}{4} \delta \theta(2 \varrho)^{2}, s+(\delta+L \bar{\delta}) \theta(2 \varrho)^{2}\right] .
$$

The quantitative information as in (4.6) will reach the top of $Q_{\varrho}(\theta)$ when $L$ is so large that $4(\delta+L \bar{\delta}) \geq 1$. Therefore, we may conclude with $\eta=\bar{\xi}^{L} \xi$.

\section{Proof of Theorem 1.1}

With all the ingredients prepared so far, we are ready to prove Theorem 1.1. The proof splits into two parts according to either $m>1$ or $0<m<1$. In the first part, we will work with cylinders of the type $Q_{\varrho}(\theta)$, whereas in the second part we use cylinders of the type $\mathcal{Q}_{\varrho}(\vartheta)$.

\subsection{When $m>1$}

We may assume that $\left(x_{o}, t_{o}\right)$ coincides with the origin and set

$$
2 M:=\max _{1 \leq i \leq n}\left\{\underset{E_{T}}{\operatorname{ess} \sup \left|u_{i}\right|}\right\} .
$$

Constructing the cylinder $Q_{\varrho}(\theta)$ with $\theta=M^{1-m}$, we may assume

$$
Q_{\varrho}(\theta) \subset E_{T} \quad \text { and } \quad \underset{Q_{\varrho}(\theta)}{\operatorname{ess} \sup }\left|u_{i}\right| \leq 2 M \quad \text { for all } i=1, \cdots, n .
$$




\subsubsection{Reduction of oscillation near zero}

Let us first suppose (4.1) holds for all $i=1, \cdots, n$. Then according to (4.2), we have

$$
\underset{Q_{\frac{\rho}{2}}(\nu \theta)}{\operatorname{ess} \sup }\left|u_{i}\right| \leq 2 M(1-\eta), \quad \text { for all } i=1, \cdots, n .
$$

If we introduce

$$
Q_{1}=Q_{\varrho_{1}}\left(\theta_{1}\right) \quad \text { with } \varrho_{1}=c \varrho, \quad c=\frac{1}{2} v^{\frac{1}{2}}(1-\eta)^{\frac{m-1}{2}}, \quad \theta_{1}=[M(1-\eta)]^{1-m},
$$

then the above estimate yields that

$$
Q_{1} \subset Q_{\frac{\varrho}{2}}(\nu \theta) \quad \text { and } \quad \operatorname{ess} \sup \left|u_{i}\right| \leq 2 M(1-\eta), \quad \text { for all } i=1, \cdots, n .
$$

Now we may proceed by induction. Suppose $\varrho_{o}=\varrho, M_{o}=M$, and up to $j=1, \cdots, \ell-1$ we have built

$$
\left\{\begin{array}{c}
\varrho_{j}=c \varrho_{j-1}, \quad M_{j}=(1-\eta) M_{j-1}, \quad \theta_{j}=M_{j}^{1-m} \\
Q_{j}=K_{\varrho_{j}} \times\left(-\varrho_{j}^{2} \theta_{j}, 0\right], \quad \max _{1 \leq i \leq n} \underset{Q_{j}}{\operatorname{ess} \sup }\left|u_{i}\right| \leq 2 M_{j}
\end{array}\right.
$$

For all the indices $j=1, \cdots, \ell-1$, we alway assume that (4.1) holds for all $i=1, \cdots, n$, i.e.,

$$
\left|\left[u_{i} \leq M_{j}\right] \cap Q_{j}\right|>v\left|Q_{j}\right| \text { and }\left|\left[u_{i} \geq-M_{j}\right] \cap Q_{j}\right|>v\left|Q_{j}\right| .
$$

In this way the previous argument can be repeated and we have for all $j=1, \cdots, \ell$,

$$
\max _{1 \leq i \leq n} \underset{Q_{j}}{\operatorname{ess} \sup }\left|u_{i}\right| \leq 2 M_{j-1}(1-\eta)=2 M_{j}
$$

Consequently, iterating the above recursive inequality we obtain for all $j=1,2, \cdots, \ell$,

$$
\max _{1 \leq i \leq n} \underset{Q_{j}}{\operatorname{ess} \sup }\left|u_{i}\right| \leq 2 M(1-\eta)^{j}=2 M\left(\frac{\varrho_{j}}{\varrho}\right)^{\alpha_{o}} \quad \text { where } \alpha_{o}=\frac{\ln (1-\eta)}{\ln c} .
$$

\subsubsection{Reduction of oscillation away from zero}

Let us suppose $\ell$ is the first index such that (4.1) is violated, that is, for some $i \in\{1, \cdots, n\}$ there holds

$$
\text { either }\left|\left[u_{i} \leq M_{\ell}\right] \cap Q_{\ell}\right| \leq v\left|Q_{\ell}\right| \text { or } \quad\left|\left[u_{i} \geq-M_{\ell}\right] \cap Q_{\ell}\right| \leq v\left|Q_{\ell}\right| .
$$

According to Lemma 3.1, we must have

$$
\text { either } u_{i} \geq \frac{1}{2} M_{\ell} \quad \text { or } \quad u_{i} \leq-\frac{1}{2} M_{\ell} \text { a.e. in } Q_{\frac{1}{2} \varrho_{\ell}}\left(\theta_{\ell}\right) \text {. }
$$

In either case we end up with

$$
|\mathbf{u}| \geq\left|u_{i}\right| \geq \frac{1}{2} M_{\ell} \quad \text { a.e. in } Q_{\frac{1}{2} \varrho_{\ell}}\left(\theta_{\ell}\right) .
$$

On the other hand, taking (5.1) for $j=\ell$ into consideration, we obtain

$$
\underset{Q_{\ell}}{\operatorname{ess} \sup }|\mathbf{u}| \leq n^{\frac{1}{2}} \max _{1 \leq i \leq n} \underset{Q_{\ell}}{\operatorname{ess} \sup }\left|u_{i}\right| \leq 2 n^{\frac{1}{2}} M_{\ell} .
$$


The lower bound (5.3) and the upper bound (5.4) of $\mathbf{u}$ permit us to realize the oscillation decay of $\mathbf{u}$ by an appeal to the classical parabolic theory in [12]. In fact, we may introduce new variables

$$
\tilde{x}=\frac{x}{\varrho_{\ell}}, \quad \tilde{t}=\frac{t}{M_{\ell}^{1-m} \varrho_{\ell}^{2}},
$$

and a new function

$$
\mathbf{w}(\tilde{x}, \tilde{t}):=M_{\ell}^{-1} \mathbf{u}\left(\varrho_{\ell} \tilde{x}, M_{\ell}^{1-m} \varrho_{\ell}^{2} \tilde{t}\right) \text { for }(\tilde{x}, \tilde{t}) \in Q_{\frac{1}{2}} .
$$

As a result, the function $\mathbf{w}$ satisfies

$$
\left\{\begin{array}{l}
\partial_{\tilde{t}} w_{i}-\operatorname{div}_{\tilde{x}} \tilde{\mathbf{A}}^{(i)}\left(\tilde{x}, \tilde{t}, \mathbf{w}, D_{\tilde{x}} \mathbf{w}\right)=0 \quad \text { weakly in } Q_{\frac{1}{2}}, \\
i=1, \cdots, n,
\end{array}\right.
$$

where the functions

$$
\widetilde{\mathbf{A}}^{(i)}\left(\tilde{x}, \tilde{t}, \mathbf{w}, D_{\tilde{x}} \mathbf{w}\right):=M_{\ell}^{-m} \varrho_{\ell} \mathbf{A}^{(i)}(x, t, \mathbf{u}, D \mathbf{u})
$$

satisfy the structure conditions

$$
\left\{\begin{array}{l}
\widetilde{\mathbf{A}}^{(i)}\left(\tilde{x}, \tilde{t}, \mathbf{w}, D_{\tilde{x}} \mathbf{w}\right) \cdot D_{\tilde{x}} w_{i} \geq C_{o}|\mathbf{w}|^{m-1}\left|D_{\tilde{x}} w_{i}\right|^{2}, \\
\left|\widetilde{\mathbf{A}}^{(i)}\left(\tilde{x}, \tilde{t}, \mathbf{w}, D_{\tilde{x}} \mathbf{w}\right)\right| \leq C_{1}|\mathbf{w}|^{m-1}\left|D_{\tilde{x}} w_{i}\right| .
\end{array}\right.
$$

Appealing to (5.3) - (5.4), we have $\frac{1}{2} \leq|\mathbf{w}| \leq 2 n^{\frac{1}{2}}$ in $Q_{\frac{1}{2}}$. This becomes a non-degenerate, diagonal system about $\mathbf{w}$. According to [12], there exist $\alpha_{1} \in(0,1)$ and $\gamma>1$ depending only on the data, such that for all $0<r<\frac{1}{2}$,

$$
\max _{1 \leq i \leq n} \underset{Q_{r}}{\operatorname{ess}} \operatorname{osc} w_{i} \leq \gamma r^{\alpha_{1}}
$$

Rephrasing this oscillation decay in terms of $\mathbf{u}$, we have for all $0<r<\varrho_{\ell}$,

$$
\max _{1 \leq i \leq n} \underset{Q_{r}\left(\theta_{\ell}\right)}{\operatorname{ess} \operatorname{osc}} u_{i} \leq \gamma M_{\ell}\left(\frac{r}{\varrho_{\ell}}\right)^{\alpha_{1}} .
$$

Combining (5.2) and (5.5), we arrive at the desired conclusion, i.e., for all $0<r<\varrho$ we have

$$
\max _{1 \leq i \leq n} \underset{Q_{r}(\theta)}{\operatorname{ess} \operatorname{osc}} u_{i} \leq \gamma M\left(\frac{r}{\varrho}\right)^{\alpha} \quad \text { where } \alpha=\min \left\{\alpha_{o}, \alpha_{1}\right\} .
$$

Here we have used $m>1$ such that $\theta \leq \theta_{\ell}$, and thus $Q_{r}(\theta) \subset Q_{r}\left(\theta_{\ell}\right)$.

\subsection{When $0<m<1$}

We set up $M$ just like in Sect. 5.1. Constructing the cylinder $\mathcal{Q}_{\varrho}(\vartheta)$ with $\vartheta:=M^{\frac{m-1}{2}}$, we may assume

$$
\mathcal{Q}_{\varrho}(\vartheta) \subset E_{T} \quad \text { and } \quad \underset{\mathcal{Q}_{\varrho}(\vartheta)}{\operatorname{ess} \sup }\left|u_{i}\right| \leq 2 M \quad \text { for all } i=1, \cdots, n .
$$




\subsubsection{Reduction of oscillation near zero}

Let us first suppose the following holds for all $i=1, \cdots, n$ :

$$
\left|\left[u_{i} \leq M\right] \cap \mathcal{Q}_{\varrho}(\vartheta)\right|>v\left|\mathcal{Q}_{\varrho}(\vartheta)\right| \text { and }\left|\left[u_{i} \geq-M\right] \cap \mathcal{Q}_{\varrho}(\vartheta)\right|>v\left|\mathcal{Q}_{\varrho}(\vartheta)\right| .
$$

Then according to Remark 4.1, we have

$$
\begin{aligned}
& \text { ess sup }\left|u_{i}\right| \leq 2 M(1-\eta), \quad \text { for all } i=1, \cdots, n . \\
& \mathcal{Q}_{\frac{\rho}{2}}\left(v^{-\frac{1}{2}} \vartheta\right)
\end{aligned}
$$

If we introduce

$$
\mathcal{Q}_{1}=\mathcal{Q}_{\varrho_{1}}\left(\vartheta_{1}\right) \quad \text { with } \varrho_{1}=c \varrho, c=\min \left\{\frac{1}{2}, \frac{1}{2} v^{-\frac{1}{2}}(1-\eta)^{\frac{1-m}{2}}\right\}, \quad \vartheta_{1}=[M(1-\eta)]^{\frac{m-1}{2}},
$$

then the above estimate yields that

$$
\mathcal{Q}_{1} \subset \mathcal{Q}_{\frac{\varrho}{2}}\left(v^{-\frac{1}{2}} \vartheta\right) \quad \text { and } \quad \underset{\mathcal{Q}_{1}}{\operatorname{ess} \sup }\left|u_{i}\right| \leq 2 M(1-\eta), \quad \text { for all } i=1, \cdots, n .
$$

Now we may proceed by induction. Suppose $\varrho_{o}=\varrho, M_{o}=M$, and up to $j=1, \cdots, \ell-1$ we have built

$$
\left\{\begin{array}{c}
\varrho_{j}=c \varrho_{j-1}, \quad M_{j}=(1-\eta) M_{j-1}, \quad \vartheta_{j}=M_{j}^{\frac{m-1}{2}} \\
\mathcal{Q}_{j}=K_{\vartheta_{j} \varrho_{j}} \times\left(-\varrho_{j}^{2}, 0\right], \quad \max _{1 \leq i \leq n} \underset{\mathcal{Q}_{j}}{\operatorname{ess} \sup \left|u_{i}\right| \leq 2 M_{j}}
\end{array}\right.
$$

For all the indices $j=1, \cdots, \ell-1$, we alway assume that for all $i=1, \cdots, n$,

$$
\left|\left[u_{i} \leq M_{j}\right] \cap \mathcal{Q}_{j}\right|>v\left|\mathcal{Q}_{j}\right| \text { and }\left|\left[u_{i} \geq-M_{j}\right] \cap \mathcal{Q}_{j}\right|>v\left|\mathcal{Q}_{j}\right| .
$$

In this way the previous argument can be repeated and we have for all $j=1, \cdots, \ell$,

$$
\max _{1 \leq i \leq n} \operatorname{ess} \sup \left|u_{i}\right| \leq 2 M_{j-1}(1-\eta)=2 M_{j}
$$

Consequently, iterating the above recursive inequality we obtain for all $j=1, \cdots, \ell$,

$$
\max _{1 \leq i \leq n} \underset{\mathcal{Q}_{j}}{\operatorname{ess} \sup }\left|u_{i}\right| \leq 2 M(1-\eta)^{j}=2 M\left(\frac{\varrho_{j}}{\varrho}\right)^{\alpha_{o}} \quad \text { where } \alpha_{o}=\frac{\ln (1-\eta)}{\ln c} .
$$

\subsubsection{Reduction of oscillation away from zero}

Let us suppose $\ell$ is the first index such that (5.6) is violated, that is, for some $i \in\{1, \cdots, n\}$ there holds

$$
\text { either }\left|\left[u_{i} \leq M_{\ell}\right] \cap \mathcal{Q}_{\ell}\right| \leq v\left|\mathcal{Q}_{\ell}\right| \quad \text { or } \quad\left|\left[u_{i} \geq-M_{\ell}\right] \cap \mathcal{Q}_{\ell}\right| \leq v\left|\mathcal{Q}_{\ell}\right| .
$$

According to Lemma 3.1, we must have

$$
\text { either } u_{i} \geq \frac{1}{2} M_{\ell} \text { or } u_{i} \leq-\frac{1}{2} M_{\ell} \text { a.e. in } \mathcal{Q}_{\frac{1}{2} \varrho_{\ell}}\left(\vartheta_{\ell}\right) \text {. }
$$

In either case we end up with

$$
|\mathbf{u}| \geq\left|u_{i}\right| \geq \frac{1}{2} M_{\ell} \quad \text { a.e. in } \mathcal{Q}_{\frac{1}{2} \varrho_{\ell}}\left(\theta_{\ell}\right) .
$$


On the other hand, taking (5.7) for $j=\ell$ into consideration, we obtain

$$
\underset{\mathcal{Q}_{\ell}}{\operatorname{ess} \sup }|\mathbf{u}| \leq n^{\frac{1}{2}} \max _{1 \leq i \leq n} \underset{\mathcal{Q}_{\ell}}{\operatorname{ess} \sup }\left|u_{i}\right| \leq 2 n^{\frac{1}{2}} M_{\ell} .
$$

Next, we may introduce new variables

$$
\tilde{x}=\frac{x}{\varrho_{\ell} M_{\ell}^{\frac{m-1}{2}}}, \quad \tilde{t}=\frac{t}{\varrho_{\ell}^{2}},
$$

and a new function

$$
\mathbf{w}(\tilde{x}, \tilde{t}):=M_{\ell}^{-1} \mathbf{u}\left(\varrho_{\ell} M_{\ell}^{\frac{m-1}{2}} \tilde{x}, \varrho_{\ell}^{2} \tilde{t}\right) \quad \text { for }(\tilde{x}, \tilde{t}) \in \mathcal{Q}_{\frac{1}{2}} .
$$

As a result, the function $\mathbf{w}$ satisfies

$$
\left\{\begin{array}{l}
\partial_{\tilde{t}} w_{i}-\operatorname{div}_{\tilde{x}} \tilde{\mathbf{A}}^{(i)}\left(\tilde{x}, \tilde{t}, \mathbf{w}, D_{\tilde{x}} \mathbf{w}\right)=0 \quad \text { weakly in } \mathcal{Q}_{\frac{1}{2}}, \\
i=1, \cdots, n
\end{array}\right.
$$

where the functions

$$
\widetilde{\mathbf{A}}^{(i)}\left(\tilde{x}, \tilde{t}, \mathbf{w}, D_{\tilde{x}} \mathbf{w}\right):=M_{\ell}^{-\frac{m+1}{2}} \varrho_{\ell} \mathbf{A}^{(i)}(x, t, \mathbf{u}, D \mathbf{u})
$$

satisfy the structure conditions

$$
\left\{\begin{array}{l}
\widetilde{\mathbf{A}}^{(i)}\left(\tilde{x}, \tilde{t}, \mathbf{w}, D_{\tilde{x}} \mathbf{w}\right) \cdot D w_{i} \geq C_{o}|\mathbf{w}|^{m-1}\left|D_{\tilde{x}} w_{i}\right|^{2}, \\
\left|\widetilde{\mathbf{A}}^{(i)}\left(\tilde{x}, \tilde{t}, \mathbf{w}, D_{\tilde{x}} \mathbf{w}\right)\right| \leq C_{1}|\mathbf{w}|^{m-1}\left|D_{\tilde{x}} w_{i}\right| .
\end{array}\right.
$$

Appealing to (5.9) - (5.10), we have $\frac{1}{2} \leq|\mathbf{w}| \leq 2 n^{\frac{1}{2}}$ in $\mathcal{Q}_{\frac{1}{2}}$. This becomes a diagonal system about $\mathbf{w}$. According to [12], there exist $\alpha_{1} \in(0,1)$ and $\gamma>1$ depending only on the data, such that for all $0<r<\frac{1}{2}$,

$$
\max _{1 \leq i \leq n} \underset{\mathcal{Q}_{r}}{\operatorname{ess}} \operatorname{osc} w_{i} \leq \gamma r^{\alpha_{1}}
$$

Rephrasing this oscillation decay in terms of $\mathbf{u}$, we have for all $0<r<\varrho_{\ell}$,

$$
\max _{1 \leq i \leq n} \underset{\mathcal{Q}_{r}\left(\vartheta_{\ell}\right)}{\operatorname{ess}} u_{i} \leq \gamma M_{\ell}\left(\frac{r}{\varrho_{\ell}}\right)^{\alpha_{1}} .
$$

Combining (5.8) and (5.11), we arrive at the desired conclusion, i.e., for all $0<r<\varrho$ we have

$$
\max _{1 \leq i \leq n} \underset{\mathcal{Q}_{r}(\vartheta)}{\operatorname{ess} \operatorname{osc}} u_{i} \leq \gamma M\left(\frac{r}{\varrho}\right)^{\alpha} \quad \text { where } \alpha=\min \left\{\alpha_{o}, \alpha_{1}\right\} .
$$

Here we have used $0<m<1$ such that $\vartheta \leq \vartheta_{\ell}$, and thus $\mathcal{Q}_{r}(\vartheta) \subset \mathcal{Q}_{r}\left(\vartheta_{\ell}\right)$.

\section{Local boundedness of solutions}

In this section, we propose some sufficient conditions that imply the local boundedness of solutions. In the following we still consider the general system (1.1). However, we need to impose more structure conditions, namely 


$$
\left\{\begin{array}{l}
\mathbf{A}^{(i)}(x, t, \mathbf{u}, D \mathbf{u}) \cdot D u_{i} \geq C_{o}|\mathbf{u}|^{m-1}\left|D u_{i}\right|^{2}, \\
\sum_{i=1}^{n} \mathbf{A}^{(i)}(x, t, \mathbf{u}, D \mathbf{u}) \cdot u_{i} D|\mathbf{u}| \geq C_{o}|\mathbf{u}|^{m}|D| \mathbf{u}||^{2}, \\
\left|\mathbf{A}^{(i)}(x, t, \mathbf{u}, D \mathbf{u})\right| \leq C_{1}|\mathbf{u}|^{m-1}\left|D u_{i}\right|
\end{array}\right.
$$

for all $i=1, \cdots, n$ and for some positive $m, C_{o}$ and $C_{1}$. Note that $(6.1)_{2}$ is additional comparing with (1.2). Nevertheless they are all verified by the prototype system (1.1).

For $m>0$, a vector valued function $\mathbf{u}$ satisfying

$$
u_{i} \in C\left(0, T ; L_{\mathrm{loc}}^{2}(E)\right),|\mathbf{u}|^{m-1} u_{i}, u_{i} \in L_{\mathrm{loc}}^{2}\left(0, T ; W_{\mathrm{loc}}^{1,2}(E)\right),
$$

is a local, weak sub(super)-solution to (1.1) with the structure conditions in (6.1), if for every compact set $K \subset E$ and every sub-interval $\left[t_{1}, t_{2}\right] \subset(0, T]$, the integral formulation (1.3) holds.

We have the following result concerning quantitative supreme estimates of local weak solutions, whose proof we use Moser's iteration to present.

Theorem 6.1 Let $\mathbf{u}$ be a local weak solution to (1.1) in $E_{T}$ with conditions (6.1). There exists $\gamma>1$ depending only on the data, such that for any $\sigma \in(0,1)$ and $\left(x_{o}, t_{o}\right)+Q_{\varrho, \tau} \subset E_{T}$, for $m>1$, we have

$$
\operatorname{ess~sup}_{\left(x_{o}, t_{o}\right)+Q_{\sigma \varrho, \sigma \tau}}|\mathbf{u}| \leq \frac{\gamma \mathcal{A}}{(1-\sigma)^{\frac{N^{2} \kappa}{4}}}\left[\frac{1}{\left|Q_{\varrho, \tau}\right|} \iint_{\left(x_{o}, t_{o}\right)+Q_{\varrho, \tau}}\left(|\mathbf{u}|^{m+1}+1\right) \mathrm{d} x \mathrm{~d} t\right]^{\frac{1}{2}},
$$

where $\mathcal{A}$ is defined in (6.6) and $\kappa:=\frac{N+2}{N}$; whereas for $0<m<1$, requiring that $\lambda:=$ $N(m-1)+2 r>0$ for some $r \geq 2$ and $\mathbf{u} \in L_{\mathrm{loc}}^{r}\left(E_{T}\right)$, we have

$$
\operatorname{ess}_{\left(x_{o}, t_{o}\right)+Q_{\sigma \varrho, \sigma \tau}}|\mathbf{u}| \leq \frac{\gamma \widetilde{\mathcal{A}}}{(1-\sigma)^{\frac{N^{2} \kappa}{\lambda}}}\left[\frac{1}{\left|Q_{\varrho, \tau}\right|} \iint_{\left(x_{o}, t_{o}\right)+Q_{\varrho, \tau}}\left(|\mathbf{u}|^{r}+1\right) \mathrm{d} x \mathrm{~d} t\right]^{\frac{2}{\lambda}}
$$

where $\widetilde{\mathcal{A}}$ is defined in (6.7).

\subsection{Energy estimates}

We first present an energy estimate that will serve as the starting point of Moser's iteration scheme.

Proposition 6.1 Let $\mathbf{u}$ be a local weak solution to (1.1) in $E_{T}$ with conditions in (6.1). Set $f(\cdot)$ to be a non-negative, non-decreasing, locally Lipschitz function in $\mathbb{R}_{+}$. There exists a constant $\gamma>0$, such that for all cylinders $Q_{R, S}:=K_{R}\left(x_{o}\right) \times\left(t_{o}-S, t_{o}\right] \subset E_{T}$, and every non-negative, piecewise smooth cutoff function $\zeta$ in $Q_{R, S}$ vanishing on $\partial K_{R}\left(x_{o}\right)$, 


$$
\begin{aligned}
& \underset{t_{o}-S<t<t_{o}}{\operatorname{ess} \sup _{K_{R}\left(x_{o}\right)}} \int_{0}^{|\mathbf{u}|} s f(s) d s \zeta^{2}(x, t) \mathrm{d} x+\iint_{Q_{R, S}}|\mathbf{u}|^{m-1}|D \mathbf{u}|^{2} f(|\mathbf{u}|) \zeta^{2} \mathrm{~d} x \mathrm{~d} t \\
& \quad+\iint_{Q_{R, S}}|\mathbf{u}|^{m}|D| \mathbf{u}||^{2} f^{\prime}(|\mathbf{u}|) \zeta^{2} \mathrm{~d} x \mathrm{~d} t \\
& \quad \leq \gamma \iint_{Q_{R, S}}|D \zeta|^{2}|\mathbf{u}|^{m+1} f(|\mathbf{u}|) \mathrm{d} x \mathrm{~d} t+\gamma \iint_{Q_{R, S}} \int_{0}^{|\mathbf{u}|} s f(s) d s\left|\zeta_{t}\right| \mathrm{d} x \mathrm{~d} t \\
& \quad+\int_{K_{R}\left(x_{o}\right)} \int_{0}^{|\mathbf{u}|} s f(s) d s \zeta^{2}\left(x, t_{o}-S\right) \mathrm{d} x,
\end{aligned}
$$

provided

$$
|\mathbf{u}|^{m+1} f(|\mathbf{u}|) \text { and } \int_{0}^{|\mathbf{u}|} s f(s) \mathrm{d} s \in L_{\mathrm{loc}}^{1}\left(E_{T}\right) .
$$

Proof Define the truncated version of $f$ by $f_{k}:=\min \{f, k\}$ for some $k>0$. Let $\psi_{\varepsilon}$ be as in Proposition 2.1. We take $\phi:=\llbracket u_{i} \rrbracket_{h} f_{k}\left(\left|\llbracket \mathbf{u} \rrbracket_{h}\right|\right) \psi_{\varepsilon} \zeta^{2}$ as a test function against the $i$-th equation of (I) in $Q:=K_{R}\left(x_{o}\right) \times\left(t_{o}-S, \tau\right]$ for $\tau<t_{o}$. In the following we omit the reference to $x_{o}$ and sum over the index $i \in\{1, \cdots, n\}$ tacitly. As in Proposition 2.1, the right-hand side of (1.5) vanishes as $h \rightarrow 0$. The time part is calculated as

$$
\begin{aligned}
& \iint_{Q} \partial_{t} \llbracket u_{i} \rrbracket_{h} \llbracket u_{i} \rrbracket_{h} f_{k}\left(\left|\llbracket \mathbf{u} \rrbracket_{h}\right|\right) \zeta^{2} \mathrm{~d} x \mathrm{~d} t \\
& \quad=\frac{1}{2} \iint_{Q} \partial_{t}\left|\llbracket \mathbf{u} \rrbracket_{h}\right|^{2} f_{k}\left(\left|\llbracket \mathbf{u} \rrbracket_{h}\right|\right) \zeta^{2} \mathrm{~d} x \mathrm{~d} t=\iint_{Q} \partial_{t} \int_{0}^{\left|\llbracket \mathbf{u} \rrbracket_{h}\right|} s f_{k}(s) \mathrm{d} s \zeta^{2} \mathrm{~d} x \mathrm{~d} t \\
& \quad=\left.\int_{K_{R}} \int_{0}^{\left|\llbracket \mathbf{u} \rrbracket_{h}\right|} s f_{k}(s) \mathrm{d} s \zeta^{2} \mathrm{~d} x\right|_{t_{o}-S} ^{\tau}-2 \iint_{Q} \int_{0}^{\left|\llbracket \mathbf{u} \rrbracket_{h}\right|} s f_{k}(s) \mathrm{d} s \zeta \zeta_{t} \mathrm{~d} x \mathrm{~d} t \\
& \left.\quad \rightarrow \int_{K_{R}} \int_{0}^{|\mathbf{u}|} s f_{k}(s) \mathrm{d} s \zeta^{2} \mathrm{~d} x\right|_{t_{o}-S} ^{\tau}-2 \iint_{Q} \int_{0}^{|\mathbf{u}|} s f_{k}(s) \mathrm{d} s \zeta \zeta_{t} \mathrm{~d} x \mathrm{~d} t \quad \text { as } h \rightarrow 0 .
\end{aligned}
$$

The space part can be calculated by sending $h \rightarrow 0$ :

$$
\begin{aligned}
& \iint_{Q} \llbracket \mathbf{A}^{(i)}(x, t, \mathbf{u}, D \mathbf{u}) \rrbracket_{h} \cdot D\left[\llbracket u_{i} \rrbracket_{h} f_{k}\left(\left|\llbracket \mathbf{u} \rrbracket_{h}\right|\right) \zeta^{2}\right] \mathrm{d} x \mathrm{~d} t \\
& \quad \rightarrow \iint_{Q} \mathbf{A}^{(i)}(x, t, \mathbf{u}, D \mathbf{u}) \cdot\left[D u_{i} f_{k}(|\mathbf{u}|) \zeta^{2}\right] \mathrm{d} x \mathrm{~d} t \\
& \quad+\iint_{Q} \mathbf{A}^{(i)}(x, t, \mathbf{u}, D \mathbf{u}) \cdot\left[u_{i} D f_{k}(|\mathbf{u}|) \zeta^{2}\right] \mathrm{d} x \mathrm{~d} t \\
& \quad+2 \iint_{Q} \mathbf{A}^{(i)}(x, t, \mathbf{u}, D \mathbf{u}) \cdot\left[u_{i} f_{k}(|\mathbf{u}|) \zeta D \zeta\right] \mathrm{d} x \mathrm{~d} t \\
& =: I_{1}+I_{2}+I_{3} .
\end{aligned}
$$

Next we estimate the above three terms separately. First, according to $(6.1)_{1}$, we have

$$
I_{1} \geq C_{o} \iint_{Q}|\mathbf{u}|^{m-1}|D \mathbf{u}|^{2} f_{k}(|\mathbf{u}|) \zeta^{2} \mathrm{~d} x \mathrm{~d} t .
$$


With regard to $I_{2}$, we calculate using $(6.1)_{2}$ :

$$
\begin{aligned}
I_{2} & =\iint_{Q} \mathbf{A}^{(i)}(x, t, \mathbf{u}, D \mathbf{u}) \cdot D f_{k}(|\mathbf{u}|) \zeta^{2} \mathrm{~d} x \mathrm{~d} t \\
& \geq C_{o} \iint_{Q}|\mathbf{u}|^{m}|D| \mathbf{u}||^{2} f_{k}^{\prime}(|\mathbf{u}|) \zeta^{2} \mathrm{~d} x \mathrm{~d} t .
\end{aligned}
$$

For $I_{3}$, we estimate using $(6.1)_{3}$ and Young's inequality:

$$
\begin{aligned}
I_{3}= & 2 \iint_{Q} \mathbf{A}^{(i)}(x, t, \mathbf{u}, D \mathbf{u}) \cdot D \zeta u_{i} f_{k}(|\mathbf{u}|) \zeta \mathrm{d} x \mathrm{~d} t \\
\geq & -\frac{1}{2} C_{o} \iint_{Q}|\mathbf{u}|^{m-1}|D \mathbf{u}|^{2} f_{k}(|\mathbf{u}|) \zeta^{2} \mathrm{~d} x \mathrm{~d} t \\
& -\gamma \iint_{Q}|D \zeta|^{2}|\mathbf{u}|^{m+1} f_{k}(|\mathbf{u}|) \mathrm{d} x \mathrm{~d} t .
\end{aligned}
$$

Collecting all above estimates and sending $k \rightarrow \infty$, we may conclude.

Remark 6.1 It is not hard to see from the proof that the energy estimate in Proposition 6.1 still holds if we replace $(6.1)_{1,3}$ by the weaker conditions

$$
\left\{\begin{array}{l}
\sum_{i=1}^{n} \mathbf{A}^{(i)}(x, t, \mathbf{u}, D \mathbf{u}) \cdot D u_{i} \geq C_{o}|\mathbf{u}|^{m-1}|D \mathbf{u}|^{2}, \\
\left|\mathbf{A}^{(i)}(x, t, \mathbf{u}, D \mathbf{u})\right| \leq C_{1}|\mathbf{u}|^{m-1}|D \mathbf{u}|, \quad i=1, \cdots, n .
\end{array}\right.
$$

\subsection{Proof of Theorem 6.1 when $m>1$}

For ease of notation, we set $v:=|\mathbf{u}|$. In the energy estimate of Proposition 6.1, we take $f(v)=v^{\beta}$ for $\beta \geq 0$, apply the inequality $|D v| \leq|D \mathbf{u}|$ to the second term on the left, and dump the third term. The test function $\zeta$ is chosen to vanish on the parabolic boundary of $Q_{R, S}$ and equal 1 on $Q_{\varrho, \tau}$, such that $|D \zeta| \leq(R-\varrho)^{-1}$ and $\left|\zeta_{t}\right| \leq(S-\tau)^{-1}$. As a result, the energy estimate in Proposition 6.1 gives

$$
\begin{aligned}
& \frac{1}{\beta+1} \operatorname{ess} \sup _{-\tau<t<0} \int_{K_{\varrho} \times\{t\}} v^{2+\beta} \mathrm{d} x+\iint_{Q_{\varrho, \tau}} v^{m-1+\beta}|D v|^{2} \mathrm{~d} x \mathrm{~d} t \\
& \quad \leq \frac{\gamma}{(R-\varrho)^{2}} \iint_{Q_{R, S}} v^{m+1+\beta} \mathrm{d} x \mathrm{~d} t+\frac{\gamma}{S-\tau} \iint_{Q_{R, S}} v^{2+\beta} \mathrm{d} x \mathrm{~d} t \\
& \quad \leq\left[\frac{\gamma}{(R-\varrho)^{2}}+\frac{\gamma}{S-\tau}\right] \iint_{Q_{R, S}}\left(v^{m+1+\beta}+1\right) \mathrm{d} x \mathrm{~d} t .
\end{aligned}
$$

In the last line we have applied Young's inequality.

Written in terms of $w:=v^{\frac{m+1+\beta}{2}}$, the above estimate gives

$$
\begin{aligned}
& \frac{1}{\beta+1} \operatorname{ess~sup}_{-\tau<t<0} \int_{K_{\varrho} \times\{t\}} w^{\frac{2(2+\beta)}{m+1+\beta}} \mathrm{d} x+\frac{1}{(m+1+\beta)^{2}} \iint_{Q_{Q, \tau}}|D w|^{2} \mathrm{~d} x \mathrm{~d} t \\
& \quad \leq\left[\frac{\gamma}{(R-\varrho)^{2}}+\frac{\gamma}{S-\tau}\right] \iint_{Q_{R, S}}\left(w^{2}+1\right) \mathrm{d} x \mathrm{~d} t .
\end{aligned}
$$


This is the starting point of Moser's iteration scheme. In order to use this energy estimate properly, we introduce for $\varrho, \tau>0, \sigma \in(0,1)$ and $n=0,1, \cdots$,

$$
\left\{\begin{array}{c}
\varrho_{n}=\sigma \varrho+\frac{(1-\sigma) \varrho}{2^{n}}, \quad \tau_{n}=\sigma \tau+\frac{(1-\sigma) \tau}{2^{n}}, \\
\tilde{\varrho}_{n}=\frac{\varrho_{n}+\varrho_{n+1}}{2}, \quad \tilde{\tau}_{n}=\frac{\tau_{n}+\tau_{n+1}}{2}, \\
K_{n}=K_{\varrho_{n}}, \quad \widetilde{K}_{n}=K_{\tilde{\varrho}_{n}}, \quad Q_{n}=K_{n} \times\left(-\tau_{n}, 0\right], \quad \widetilde{Q}_{n}=\widetilde{K}_{n} \times\left(-\tilde{\tau}_{n}, 0\right], \\
p_{n}=m+1+\beta_{n}, \quad q_{n}=\frac{2\left(2+\beta_{n}\right)}{m+1+\beta_{n}}, \quad \beta_{n+1}=\kappa \beta_{n}+\frac{4}{N}, \quad \kappa=1+\frac{2}{N} .
\end{array}\right.
$$

Note that the choice of sequences in the last line implies

$$
p_{n+1}=\left(m+1+\beta_{n}\right) \frac{N+q_{n}}{N} .
$$

Now set $\zeta$ to be a standard cutoff function that vanishes on $\partial_{p} \widetilde{Q}_{n}$ and equals identity in $Q_{n+1}$, such that $|D \zeta| \leq 2^{n} / \varrho$. We apply the Sobolev imbedding (cf. [4, Chapter I, Proposition 3.1]), together with the energy estimate in $\widetilde{Q}_{n} \subset Q_{n}$ and the choice $\beta_{o}=0$, to obtain

$$
\begin{aligned}
& \iint_{Q_{n+1}} v^{p_{n+1}} \mathrm{~d} x \mathrm{~d} t \leq \iint_{\widetilde{Q}_{n}}(w \zeta)^{2 \frac{N+q_{n}}{N}} \mathrm{~d} x \mathrm{~d} t \\
& \quad \leq \gamma \iint_{\widetilde{Q}_{n}}|D(w \zeta)|^{2} \mathrm{~d} x \mathrm{~d} t\left[\underset{-\tilde{\tau}_{n}<t<0}{\left.\operatorname{ess} \sup _{\widetilde{K}_{n} \times\{t\}}(w \zeta)^{q_{n}} \mathrm{~d} x\right]^{\frac{2}{N}}}\right. \\
& \quad \leq \frac{\gamma\left(m+1+\beta_{n}\right)^{2+\frac{2}{N}}}{(1-\sigma)^{2 \kappa}}\left(\frac{2^{2 n}}{\varrho^{2}}+\frac{2^{n}}{\tau}\right)^{\kappa}\left[\iint_{Q_{n}}\left(v^{p_{n}}+1\right) \mathrm{d} x \mathrm{~d} t\right]^{\kappa} \\
& \quad \leq \frac{\gamma b^{n}}{(1-\sigma)^{2 \kappa}}\left(\frac{1}{\varrho^{2}}+\frac{1}{\tau}\right)^{\kappa}\left[\iint_{Q_{n}}\left(v^{p_{n}}+1\right) \mathrm{d} x \mathrm{~d} t\right]^{\kappa},
\end{aligned}
$$

for some $b, \gamma>1$ depending only on the data. To simplify the above recurrence inequality, we take the power $p_{n+1}^{-1}$ on both sides, set

$$
Y_{n}=\left[\frac{1}{\left|Q_{n}\right|} \iint_{Q_{n}}\left(v^{p_{n}}+1\right) \mathrm{d} x \mathrm{~d} t\right]^{\frac{1}{p_{n}}},
$$

and rewrite it as

$$
Y_{n+1} \leq B^{\frac{n}{p_{n+1}}} Y_{n}^{\frac{\kappa p_{n}}{p_{n+1}}}
$$

where

$$
B:=\frac{\gamma}{(1-\sigma)^{2 \kappa}}\left[\left(\frac{\tau}{\varrho^{2}}\right)^{\frac{2}{N+2}}+\left(\frac{\varrho^{2}}{\tau}\right)^{\frac{N}{N+2}}\right]^{\kappa} .
$$

Iterating this inequality yields

$$
Y_{n} \leq B^{\frac{1}{p_{n+1}}\left[n+\kappa(n-1)+\kappa^{2}(n-2)+\cdots+\kappa^{n-1}\right]} Y_{o}^{\frac{p_{o}}{p_{n+1}} \kappa^{n+1}}
$$

One calculates the limits

$$
\lim _{n \rightarrow \infty} \frac{1}{p_{n+1}} \sum_{j=0}^{n} \kappa^{j}(n-j)=\frac{N^{2}}{8}, \quad \lim _{n \rightarrow \infty} \frac{p_{o}}{p_{n+1}} \kappa^{n+1}=\frac{m+1}{2} .
$$


As a result, sending $n \rightarrow \infty$ in (6.5) gives

$$
\underset{Q_{\sigma \varrho, \sigma \tau}}{\operatorname{ess} \sup v} \leq \frac{\gamma \mathcal{A}}{(1-\sigma)^{\frac{N^{2} \kappa}{4}}}\left[\frac{1}{\left|Q_{o}\right|} \iint_{Q_{o}}\left(v^{m+1}+1\right) \mathrm{d} x \mathrm{~d} t\right]^{\frac{1}{2}},
$$

where

$$
\mathcal{A}:=\left[\left(\frac{\varrho^{2}}{\tau}\right)^{\frac{N}{N+2}}+\left(\frac{\tau}{\varrho^{2}}\right)^{\frac{2}{N+2}}\right]^{\frac{N^{2} \kappa}{8}} .
$$

\subsection{Proof of Theorem 6.1 when $0<m<1$}

The proof in this case is similar to the previous section. We indicate some key differences. First of all, the energy estimate departs from (6.2), which now becomes

$$
\begin{aligned}
& \frac{1}{\beta+1} \operatorname{ess~sup~}_{-\tau<t<0} \int_{K_{\varrho} \times\{t\}} v^{2+\beta} \mathrm{d} x+\iint_{Q_{\varrho, \tau}} v^{m-1+\beta}|D v|^{2} \mathrm{~d} x \mathrm{~d} t \\
& \quad \leq\left[\frac{\gamma}{(R-\varrho)^{2}}+\frac{\gamma}{S-\tau}\right] \iint_{Q_{R, S}}\left(v^{2+\beta}+1\right) \mathrm{d} x \mathrm{~d} t .
\end{aligned}
$$

Introduce the sequences $\varrho_{n}, \tilde{\varrho}_{n}, \tau_{n}, \tilde{\tau}_{n}, q_{n}, K_{n}, \widetilde{K}_{n}, Q_{n}, \widetilde{Q}_{n}$, and $\kappa$ as in Sect. 6.2. In this section, the sequences $p_{n}$ and $\beta_{n}$ are defined by

$$
p_{n}=2+\beta_{n}, \quad \beta_{n+1}=\kappa \beta_{n}+\frac{4}{N}+m-1, \text { such that } p_{n+1}=\left(m+1+\beta_{n}\right) \frac{N+q_{n}}{N} .
$$

Next, an application of the Sobolev imbedding as in Sect. 6.2 would yield that

$$
\iint_{Q_{n+1}} v^{p_{n+1}} \mathrm{~d} x \mathrm{~d} t \leq \frac{\gamma b^{n}}{(1-\sigma)^{2 \kappa}}\left(\frac{1}{\varrho^{2}}+\frac{1}{\tau}\right)^{\kappa}\left[\iint_{Q_{n}}\left(v^{p_{n}}+1\right) \mathrm{d} x \mathrm{~d} t\right]^{\kappa},
$$

for some $\gamma, b>1$ depending only on the data.

The sequence $\left\{\beta_{n}\right\}$ is increasing and the first number $\beta_{o}$ is chosen to ensure $p_{n+1}>p_{n}$. A simple calculation indicates this amounts to

$$
\lambda:=N(m-1)+2 r>0 \quad \text { where } r:=\beta_{o}+2 .
$$

As in Sect. 6.2, one defines $Y_{n}$ as in (6.3) and obtains a recurrence inequality as in (6.4) and (6.5). Next, one calculates the limits

$$
\lim _{n \rightarrow \infty} \frac{1}{p_{n+1}} \sum_{j=0}^{n} \kappa^{j}(n-j)=\frac{N^{2}}{2 \lambda}, \quad \lim _{n \rightarrow \infty} \frac{p_{o}}{p_{n+1}} \kappa^{n+1}=\frac{2 r}{\lambda} .
$$

Hence sending $n \rightarrow \infty$ in (6.5) gives

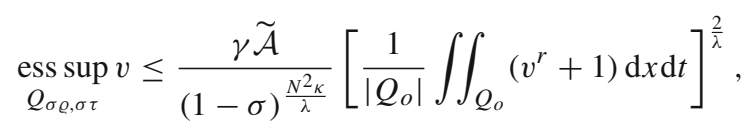

where

$$
\widetilde{\mathcal{A}}:=\left[\left(\frac{\varrho^{2}}{\tau}\right)^{\frac{N}{N+2}}+\left(\frac{\tau}{\varrho^{2}}\right)^{\frac{2}{N+2}}\right]^{\frac{N^{2} \kappa}{2 \lambda}} .
$$


Acknowledgements This research has been supported by the FWF-Project P31956-N32 "Doubly nonlinear evolution equations".

Funding Open access funding provided by Paris Lodron University of Salzburg.

Open Access This article is licensed under a Creative Commons Attribution 4.0 International License, which permits use, sharing, adaptation, distribution and reproduction in any medium or format, as long as you give appropriate credit to the original author(s) and the source, provide a link to the Creative Commons licence, and indicate if changes were made. The images or other third party material in this article are included in the article's Creative Commons licence, unless indicated otherwise in a credit line to the material. If material is not included in the article's Creative Commons licence and your intended use is not permitted by statutory regulation or exceeds the permitted use, you will need to obtain permission directly from the copyright holder. To view a copy of this licence, visit http://creativecommons.org/licenses/by/4.0/.

\section{References}

1. Bögelein, V., Lehtelä, P., Sturm, S.: Regularity of weak solutions and supersolutions to the porous medium equation. Nonlinear Anal. 185, 49-67 (2019)

2. Chen, Y.-Z.: Hölder continuity of the gradient of solutions of nonlinear degenerate parabolic systems. Acta Math. Sinica (N.S.) 2(4), 309-331 (1986)

3. Chen, Y.-Z.: Hölder continuity of the gradient of the solutions of certain degenerate parabolic equations. Chin. Ann. Math. Ser. B 8(3), 343-356 (1987)

4. DiBenedetto, E.: Degenerate Parabolic Equations. Universitext. Springer, New York (1993)

5. DiBenedetto, E.: $C^{1+\alpha}$ local regularity of weak solutions of degenerate elliptic equations. Nonlinear Anal. 7(8), 827-850 (1983)

6. DiBenedetto, E., Gianazza, U., Vespri, V.: Harnack's Inequality for Degenerate and Singular Parabolic Equations. Springer Monographs in Mathematics, Springer-Verlag, New York (2012)

7. DiBenedetto, E., Friedman, A.: Hölder estimates for nonlinear degenerate parabolic systems. J. Reine Angew. Math. 357, 1-22 (1985)

8. Giaquinta, M., Struwe, M.: On the partial regularity of weak solutions of nonlinear parabolic systems. Math. Z. 179(4), 437-451 (1982)

9. Kim, S., Lee, K.-A.: Local continuity and asymptotic behaviour of degenerate parabolic systems, Nonlinear Anal., 192, 111702, 32 pp (2020)

10. Kinnunen, J., Lindqvist, P.: Pointwise behaviour of semicontinuous supersolutions to a quasilinear parabolic equation. Ann. Mat. Pura Appl. (4) 185(3), 411-435 (2006)

11. Kuusi, T., Monsaingeon, L., Videman, J.: Systems of partial differential equations in porous medium. Nonlinear Anal. 133, 79-101 (2016)

12. Ladyzhenskaya, O.A., Solonnikov, V.A., Ural'tseva, N.N.: Linear and Quasilinear Equations of Parabolic Type, Translations of Mathematical Monographs, vol. 23. American Mathematical Society, Providence, R.I. (1968)

13. Le, D., Nguyen, T.T.: Everywhere regularity of solutions to a class of strongly coupled degenerate parabolic systems. Comm. Partial Differ. Equ. 31(1-3), 307-324 (2006)

14. Liao, N.: A unified approach to the Hölder regularity of solutions to degenerate and singular parabolic equations. J. Differ. Equ. 268(10), 5704-5750 (2020)

15. Trokhimtchouk, M.: Everywhere regularity of certain nonlinear diffusion systems. Calc. Var. Partial Differ. Equ. 37(3-4), 407-422 (2010)

16. Sanikidze, T.A., Tedeev, A.F.: On the temporal decay estimates for the degenerate parabolic system. Commun. Pure Appl. Anal. 12(4), 1755-1768 (2013)

17. Wiegner, M.: On $C_{\alpha}$-regularity of the gradient of solutions of degenerate parabolic systems. Ann. Mat. Pura Appl. 4(145), 385-405 (1986)

18. Wiegner, M.: Global solutions to a class of strongly coupled parabolic systems. Math. Ann. 292(4), 711-727 (1992)

19. Yin, H.-M.: On a degenerate parabolic system. J. Differ. Equ. 245(3), 722-736 (2008)

Publisher's Note Springer Nature remains neutral with regard to jurisdictional claims in published maps and institutional affiliations. 